\title{
Research on Simulation Calculation of the Safety of Tight-Lock Coupler Curve Coupling
}

\author{
Hai Zhang ${ }^{1,2}$, Chenyu Zhang ${ }^{2, *}$, , Fengtao Lin ${ }^{2}$, Xiugang Wang ${ }^{3}$ and Gengzhe Fu ${ }^{2}$ \\ 1 State Key Laboratory of Traction Power, Southwest Jaotong University, Chengdu 610031, China; \\ 1098@ecjtu.edu.cn \\ 2 Key Laboratory of Ministry of Education for Conveyance and Equipment, East China Jiaotong University, \\ Nanchang 330013, China; 1342@ecjtu.edu.cn (F.L.); 2020038085500080@ecjtu.edu.cn (G.F.) \\ 3 CRRC Qingdao Sifang Vehicle Co., Ltd., Qingdao 266111, China; wangxiugang@cqsf.com \\ * Correspondence: 2019038085222002@ecjtu.edu.cn or chenyuzh.v@foxmail.com
}

check for updates

Citation: Zhang, H.; Zhang, C.; Lin, F.; Wang, X.; Fu, G. Research on Simulation Calculation of the Safety of Tight-Lock Coupler Curve Coupling. Symmetry 2021, 13, 1997. https://doi.org/10.3390/ sym13111997

Academic Editor: Jan Awrejcewicz

Received: 24 September 2021

Accepted: 17 October 2021

Published: 21 October 2021

Publisher's Note: MDPI stays neutral with regard to jurisdictional claims in published maps and institutional affiliations.

Copyright: (c) 2021 by the authors. Licensee MDPI, Basel, Switzerland. This article is an open access article distributed under the terms and conditions of the Creative Commons Attribution (CC BY) license (https:/ / creativecommons.org/licenses/by/ $4.0 /)$.

\begin{abstract}
Once a train breaks down on a busy railway line, it will affect the whole traffic network. However, when a rescue locomotive is hooked up to the broken train for towing it to the next station, it is common that coupling dislocation occurs, which results in damages to couplers and the driver's cab. To ensure the safety of the trains during the coupling, it becomes crucial to determine whether they can be linked safely and automatically under different line conditions. In this paper, position and pose of the rescue locomotive and the broken train on the line are calculated by geometric analytical calculation method, which determines the position relation of their couplers. Then a so-called "coupling characteristic triangle" was proposed to determine whether trains can be safely and automatically linked on the railway line. The triangles are constructed by the peak points of the couplers head of the front vehicle and the rear one and border lines of secure coupling area on the couplers. By judging the shape of the triangle, it can directly judge whether their couplers can be connected successfully. The method has been applied to check the safety of the trains during coupling on the Nanchang urban railway Line 4 . The results show that the maximum swing angle of the coupler reaches $17.3957^{\circ}$ in the straight-curve section with a radius of $325 \mathrm{~m}$. At this time, coupling is most difficult, and trains need to be connected manually through the tractor; all the calculation results are verified in the actual line. By comparing different calculation methods for judging coupling safety, it is shown that the method proposed in this paper is accurate, efficient, and users can judge coupling safety more intuitively.
\end{abstract}

Keywords: curve link; tight-lock coupler; geometric analytic method; dynamic calculation; working conditions

\section{Introduction}

Currently, China is investing heavily in metro construction. In addition to Beijing, Shanghai, Guangzhou and Shenzhen, second-tier and third-tier cities have also started metro construction. Compared with the traditional railroad operation, the short interval and high density of the officially operated subway cars require high reliability, so the requirements for accident rescue are high and effective emergency countermeasures and plans need to be developed. When a train breaks down, trains on the same line will carry out rescue, towing the faulty train to the next station or towing it back to the yard; sometimes train transfer within the yard line also requires a train to be linked. When the subway trains are linked, there have been many safety accidents caused by not using the correct connection method, resulting in misalignment and damage of the coupler, and even damage to the driver's cab. Therefore, the study of the relative positions of the train ends and the motion of the end connection components during line operation can provide a test basis for the design of the end structure in the development of new models to prevent the interference of adjacent ends and their connection devices during train operation. 
Generally, the curve passing capacity is determined after the vehicle design and the selection of the vehicle end unit have been completed. To ensure that the curve passing capacity of the train can meet the actual operating line requirements [1], relevant calculations need to be performed during the vehicle design process [2-7]. After the vehicle has been designed and produced and delivered to the customer, to ensure the safe operation of the train, the impact of unexpected accidents on the operational efficiency of the rail transit system should be considered, and then the rescue plan should be demonstrated in accordance with the basic principles and organizational methods of fault rescue. When a vehicle breaks down, adjacent vehicles on the same route or proprietary rescue vehicles must be called in for continuous rescue for vehicles that cannot operate. The linkage between the faulty vehicle and the rescue vehicle is the key link in the whole train rescue process, in which whether the coupler connection action can be completed smoothly is very important. In straight line conditions, the connection of the couplers is accomplished by automatic collision of the two vehicles at low speeds, without manual intervention; However, if the failure occurs under curved line conditions, there is no way to ensure that the automatic hook-up range can cover the actual deflection limit of the hook, and if the personnel concerned do not find out in time that the hook cannot be automatically hooked up, there is a high probability that the hook and the end of the car will be damaged due to hook-up failure. In addition, the different selection of rescue vehicles will inevitably increase the diversity of the results of the linkage, thus creating a certain obstacle to the development of rescue programs. Therefore, it is particularly important to obtain the relative position of each part of the vehicle end during the curve rescue linkage.

Most of the existing analytical methods use Management Software Computer Aided Design to draw the relative position map of the coupler and then perform two-dimensional simulation analysis. Huang Wanchu, under the basic assumption that the center of the bogie is still in the center of the curve, made a theoretical analysis of the calculation method of the maximum hook angle when the continuous vehicle passes the geometric curve [8]. Shan Wei and Li Rui Chun derived the calculation methods for the hook angle, windshield compression and relative misalignment and end protrusions of the continuous vehicle, and prepared a programmed process for the verification of the end device [9]. Angela O. Shvets devoted to the study of the influence of the crane bogie lateral displacement on its main dynamics and indicators of interaction between the rolling stock and the track, obtained the dependence of the wagon dynamic indicators on the value of the body rotation angle and the speed of movement [10]. Zhang Guangshi et al. developed a general calculation procedure on solving the interference problem between bogies of compound-hinged trains, which can avoid tedious manual calculations and thus improve the calculation efficiency [11]. Wang Wentao et al. used the geometric parsimony method to design the position solving procedure for locomotives passing through curves, which can parametrically plot the geometric position of locomotives on the curve [12]. Wang Luke introduced the concept of "equal small radius", proposed to consider the impact of additional deflection caused by the vehicle bogie on the locomotive through the curve, and checked the hook angle and the angle of the car end wall and the limit shop distance, etc. [13]. Zhang Kun et al. explored the calibration process of the content related to the passing capacity of the vehicle end connection device of the rolling stock under horizontal and vertical curve conditions, taking the Malaysian rolling stock as an example [14]. According to the existing standards, Luo Ren et al. took the method of dynamic envelope to determine the lateral offset of the car body bogie relative to the centerline of the line, and then completed the calculation and analysis of the hook angle and windshield bending angle through the geometric curve of the attached vehicle [15].

However, most of the analysis methods are complicated, which leads to incomplete analysis of the hook attachment at different positions in different curves, and it is not easy to find the most difficult rescue attachment conditions, and it is easy to carry out the curve rescue attachment work without finding the worst conditions, which leads to the damage of the hook and the car end [16-19]. Therefore, we further consider the hook position state 
based on the vehicle position determination scheme during curve passage, and propose an algorithm scheme to determine whether the mechanical part of the hook can complete the automatic linkage during curve passage based on the whole vehicle linkage experiments conducted on the Line 4 of Nanchang Railway, and realize the development of related applications. The contributions of the paper are as follows:

1. We combined with the actual situation on the line of Nanchang Rail Transit Line 4, comprehensive consideration of the geometric offset of the vehicle, wheel-rail clearance, transverse offset of the axle and bogie, transverse offset between bogie and car body, quasi-static offset and other factors on the impact of the continuous vehicle in the operation process, to obtain the maximum transverse offset of the train in the curve rescue continuous process.

2. We propose a new method to determine the coupler position, by calculating and analyzing the trajectory characteristics of the train under different line conditions, to verify whether the front-end couplers can be connected automatically and whether the coupler swing angle meets the connection requirements, to obtain the actual connection of the couplers on the horizontal curve.

3. Our calculation method fully takes into account the effect of the lateral offset of the vehicle on the vehicle during the curve rescue linkage. Therefore, our algorithm is applicable to various line conditions, and it can accurately determine whether the hook swing angle meets the curve rescue linkage requirements when the train is under curve working conditions. Line calibration of Nanchang Rail Transit Line 3 through our calibration method, from the start of the line to date, there have been no accidents related to the rescue streak. In addition, after analyzing and judging by our calibration method under the actual line operation conditions of Nanchang Rail Transit Line 4, the parameters of some sections of the line were optimized and improved to ensure the safety of the trains in the curve rescue linkage.

4. Our calculation method fully takes into account the influence of the lateral deflection of the vehicle on the coupling of the vehicle in curve rescue. Therefore, our algorithm is applicable to various line conditions and can accurately determine whether the coupler swing angle meets the requirements for rescue coupling when the train is in curve conditions.

The rest of this paper is organized as follows. In Section 2, two popular methods of determining the hook position are described. In Section 3, the principle of Type 330 hook linkage and the main parameter setting of the analysis object is presented and the process of calculating the lateral offset of the vehicle is described. The basic principles of the calculation method are described in detail in Section 4 . The calculation results and the corresponding safety solutions are given in Section Section 5 . The conclusions are given in Section 6 .

\section{Related Works}

Research has been conducted on the longitudinal impulse of trains as part of current railway operations. However, few studies have been conducted on the calculation of train coupling, and the existing literature greatly simplifies the process of train coupling calculation without in-depth algorithm analysis, influencing factor analysis, and comparison with impact test results. The simulation calculation reports issued by Siemens, Voith, and other companies are internal reports, and there are no related algorithms or analyses available. After comprehensively considering this situation, we conducted research and an in-depth analysis of the automatic coupling of the tight-lock coupler.

In the following, we provide an overview of the two mainstream algorithms that exist today and elaborate on the shortcomings of each of them.

\subsection{CAD 2D Geometric Drawing Simulation Analysis Method}

As shown in Figure 1, the method takes the dimensions of the vehicle and the line as the basic dimensions, reproduces the vehicle end connection device in equal scale by CAD 
manual geometric drawing, and combines the actual measured offset of each wheel pair of the vehicle, treats the attached vehicle as a static mechanism to determine the center of rotation of the vehicle on the curve, and then obtains the relative positions of the vehicle and the vehicle end connection device.

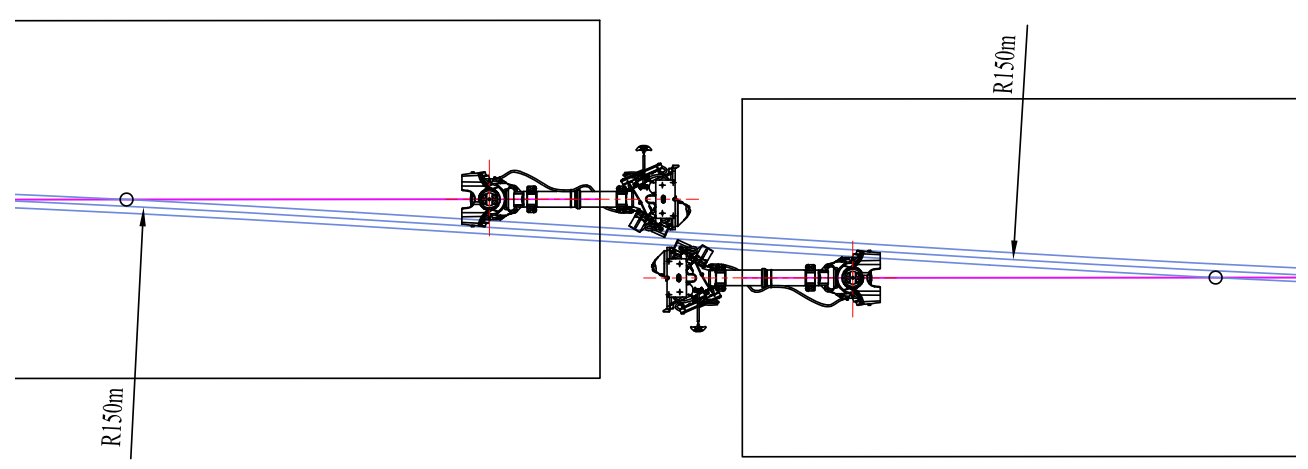

Figure 1. CAD 2D geometric drawing method.

\subsection{Analytical Method}

This method is the calculation method used by Voytek. According to the existing standards, Voytek completed the calculation and analysis of the coupling turning angle of the linked vehicle during the curve rescue linkage under the basic assumptions such as the bogie center is still in the curve center. The program is written to directly output the hook position and vehicle position as shown in Figure 2 . The image is of low definition as it is an internal material of Voytek.

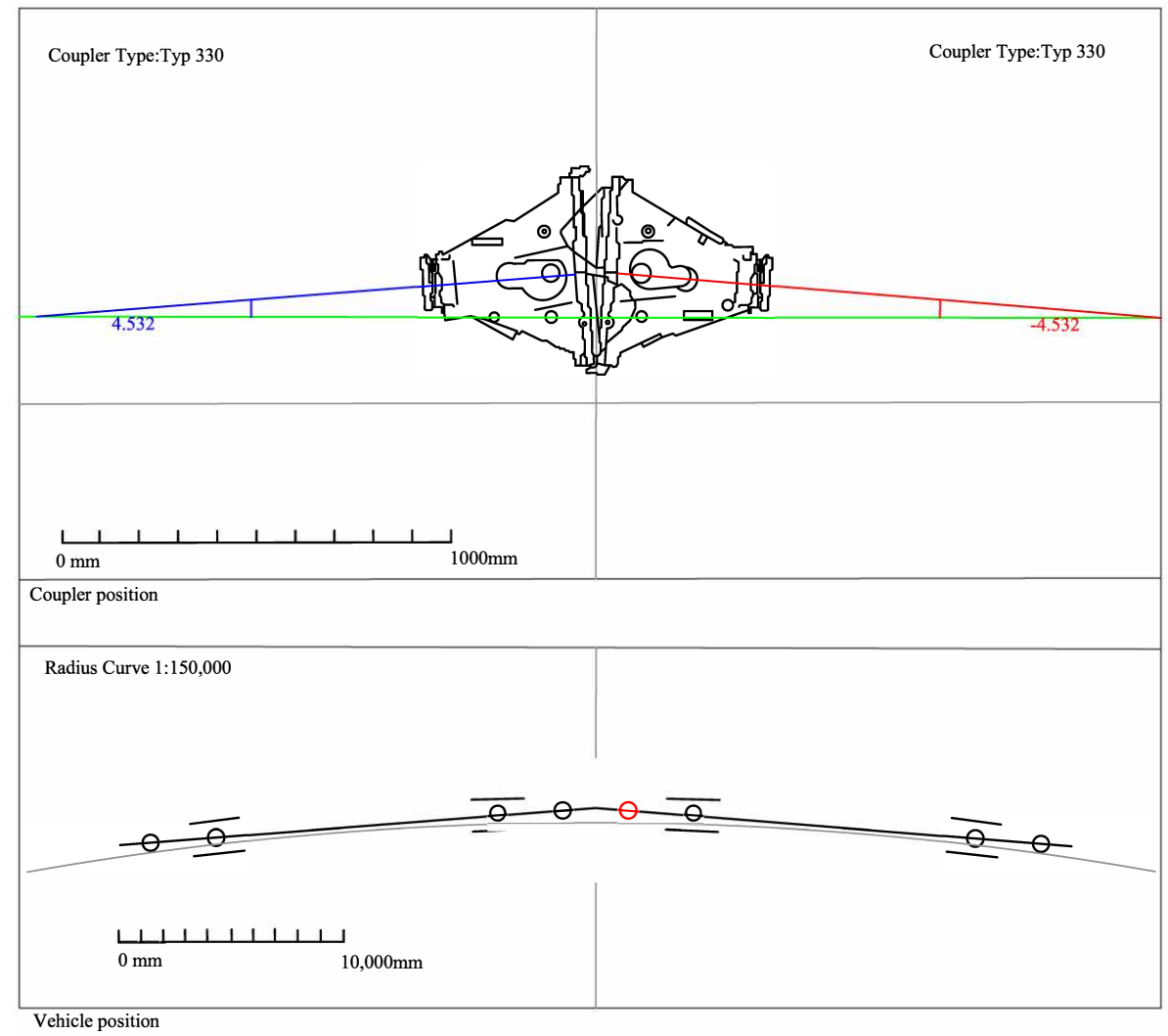

Figure 2. Schematic diagram of hook position and vehicle position. 
Although the method avoids tedious manual calculations compared with the CAD 2D geometric drawing simulation analysis method, it can greatly improve the work efficiency. However, since the lateral offset of the vehicle bogie relative to the line centerline is not taken into account in this method, the calculation results obtained by this method are not particularly accurate. In addition, this calculation method can only yield the maximum swing angle of the coupler, and does not provide a quantitative analysis of whether the coupler can be automatically linked in curves.

\section{Preliminaries}

\subsection{Structure and Principle}

Type 330 hook is a European type of hook, this hook is flexible and convenient to hang and unhook, it is a mechanical hook widely used in Chinese urban rail vehicles at present $[20,21]$. As shown in Figure 3 , the 330-type hook is mainly composed of coupler body, spindle, knuckle, coupling link, tension spring, etc. The front half of the coupler body is a convex cone, the other half is a concave cone, the connection of the two sides of the coupler convex cone and concave cone inserted into each other to play a role in the connection guide. coupler body, knuckle, coupling link, spindle, etc. are the key parts of the hook to achieve action, knuckle rotating around the spindle can drive the coupling link action, the coupling link under the action of tension spring to make a reliable connection of the coupler.

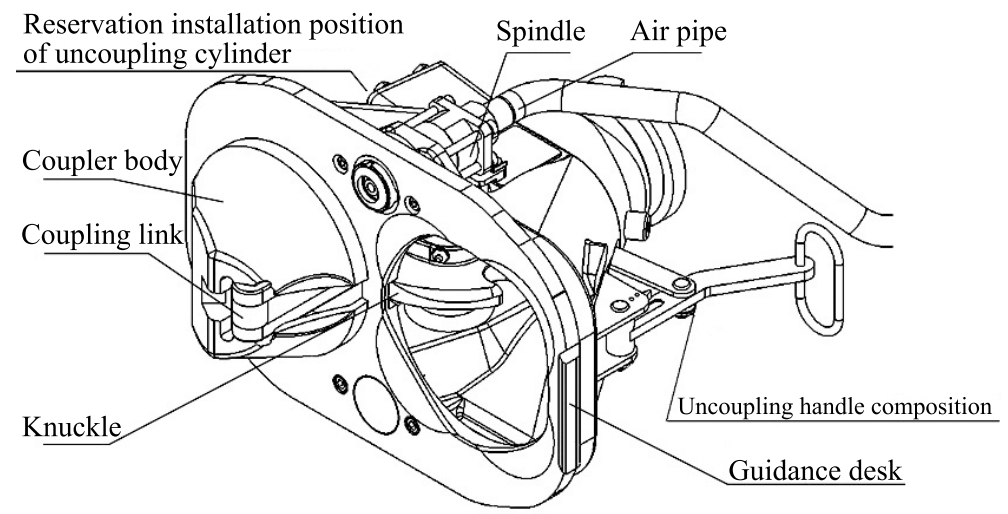

Figure 3. Basic structure of 330 type hook.

When one group of trains moves hooks to another group of trains at low speed, Type 330 hooks can realize the automatic connection of mechanical, air and electric circuits of two groups of vehicles, and the hooks can ensure a tight connection and have good adaptability; good connection between two vehicles with vertical deviation and on horizontal and vertical curves; automatic unhooking or manual unhooking can be carried out, and the hook is in the state to be connected after the unhooking of the vehicle group. Type 330 hook can be assembled with buffer system and mounting suspension system to form a hook buffer device, which is used on all kinds of newly built urban rail vehicles. The 330type hook principle of action is shown in Figure 4, with three states of pending hooking, continuous hooking and unhooking. 


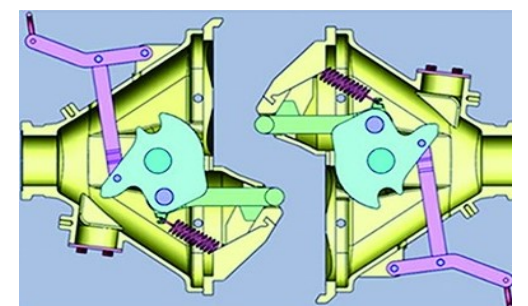

(a) Pending hooking

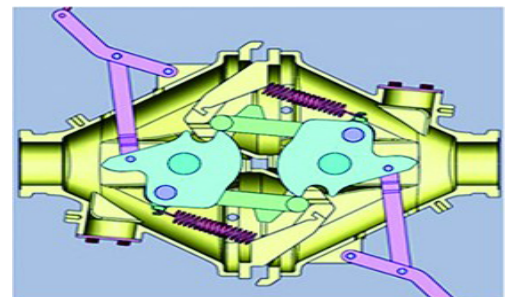

(b) Continuous hanging process1

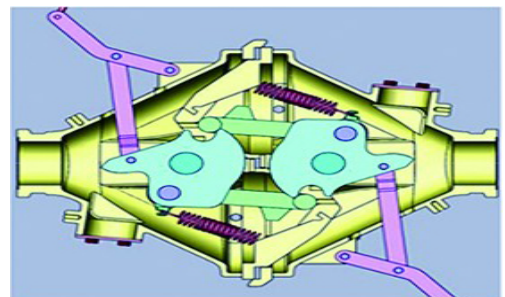

(c) Continuous hanging process2

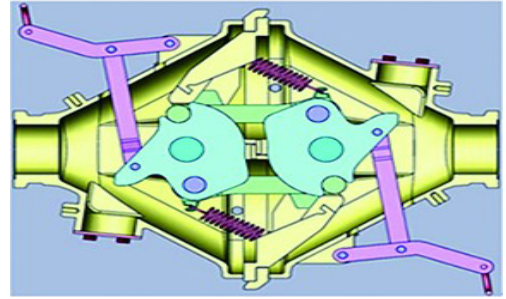

(d) Continuous hooking

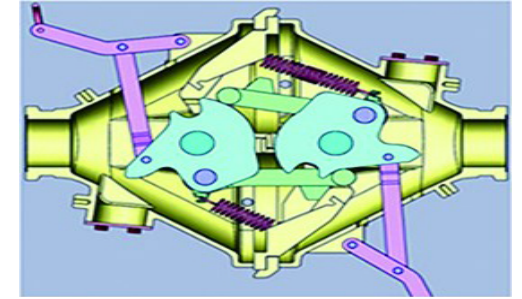

(e) Unhooking

Figure 4. The principle of 330 hook function. (a) The state of two hooks pending hooking; (b) The state of the convex cone of the connecting hook extending into the concave cone of the opposing hook; (c) Increased hook rotation angle; (d) Two hooks fully connected state; (e) The unhooked state of two car hooks.

- Pending hooking. As shown in Figure 4a, at this time, the tension spring is in the maximum tension state, the coupling link is placed in the convex cone, and the connected composition is in the pending state.

- Continuous hooking. As shown in Figure $4 b-d$, the convex cone of the connecting hook extends into the concave cone of the opposing hook, and the knuckle on both sides pushes the opposing coupling link back to cause the knuckle to rotate, and as the hooks approach each other, the angle of the knuckle rotation increases, and under the action of the tension spring, the coupling link can enter the opposing knuckle to complete the connecting of the two couplers.

- Unhooking. Manually pull one side of the hook unhooking handle, drive the knuckle clockwise rotation, when the knuckle turned to a certain angle, the knuckle of this side of the hook, when the coupling link is in the unhooked state as shown in Figure 4e, then move one side of the hook body, you can complete the decomposition of the hook. When the two hooks are separated, the coupling link is reset due to the action of the tension spring, and the hook returns to the pending state.

\subsection{Parameters}

There are three types of railway curves: plane horizontal curves, vertical curves and spiral curves [22]. In the horizontal plane, two straight lines are connected by a small arc to form a curve, referred to as a plane curve. In the vertical surface, each slope section is connected by a curve to form a vertical arch, which is called a vertical curve [23,24]. Spiral curves connect to track sections of uniform cross level (or cant). The track twist to change from level track to canted track typically for horizontal curves. Thus, most track spirals not only include changing cross level but a changing horizontal curvature and may include changing slope as well. This paper only analyzes the working conditions of the vehicles on the horizontal curve.

Take the Metro Type A car as an example, Figure 5 shows the geometric parameters of the vehicle position. Figure 6 shows the geometric characteristics of the tight-lock coupler. Table 1 shows its geometric parameters. 


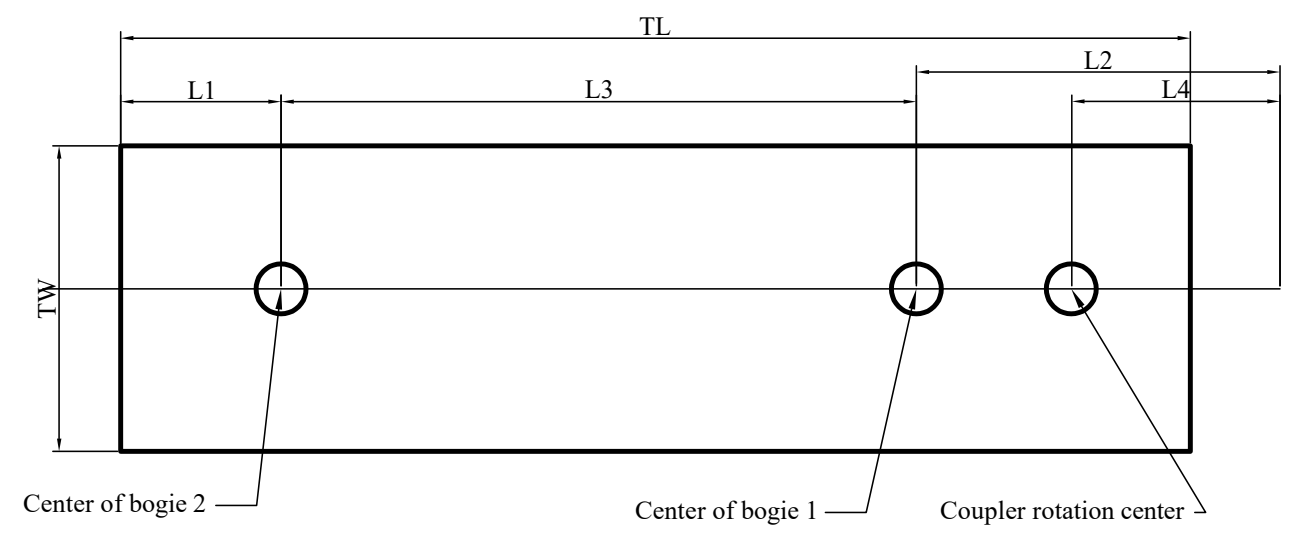

Figure 5. Schematic diagram of vehicle posture geometry parameters.

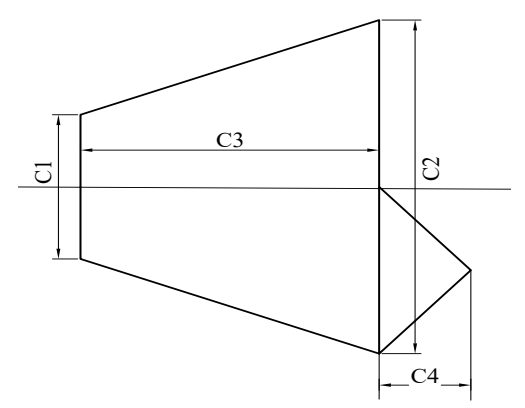

(a)

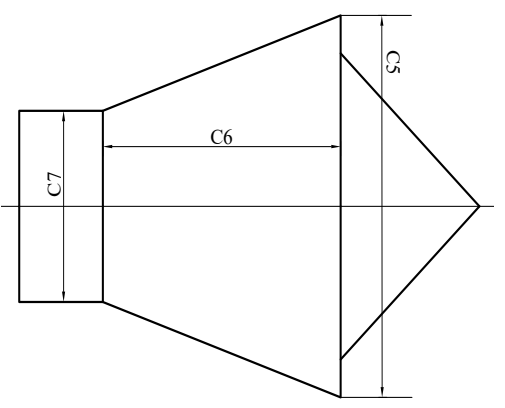

(b)

Figure 6. Schematic diagram of geometric parameters of 330 type hook. (a) Top view. (b) Main view.

Table 1. Geometric parameters table of Metro A and 330 model coupler.

\begin{tabular}{cccl}
\hline $\begin{array}{c}\text { Serial } \\
\text { Number }\end{array}$ & Parameter & Initial Value (mm) & \\
\hline 1 & L & 2100 & Remarks \\
2 & L2 & 2339 & Tistance from the center of the 2-position bogie to the vehicle end \\
3 & L3 & 12,600 & Distance from 1 bogie center to 2 bogie center \\
4 & L4 & 1225 & Distance from coupler rotation center to even noodle \\
5 & TL & 19,000 & Vehicle length \\
6 & TW & 2800 & Vehicle width \\
7 & C1 & 140 & Width of hook head and tail \\
8 & C2 & 480 & Hook head width \\
9 & C3 & 280 & Hook length \\
10 & C4 & 115 & Length of hook head convex cone \\
11 & C5 & 297 & Height of hook head convex cone (top view) \\
12 & C6 & 150 & Hook length \\
13 & C7 & 100 & Width of hook head and tail \\
14 & W1 & 170 & Horizontal left distance of hook head connecting position \\
15 & W2 & 170 & The horizontal right distance of the hook head connecting position \\
16 & W3 & 90 & The distance from the upper side of the hook head connecting position \\
17 & W4 & 90 & The distance from the lower side of the hook position \\
\hline
\end{tabular}




\subsection{Vehicle Curve through Offset Calculation}

When the vehicle passes through the curve-line, the bogie center and the center line of the line will exist a certain angle, the vehicle body due to its own transverse inertia force and produce a certain amount of transverse offset, so that the relative misalignment between the car end to reach the maximum, the risk of damage to the car end connection device. The vehicle offsets can be considered to be two components, namely the static part and the dynamic part. The static part is the offset due to gauge error and wheel-rail wear as well as vehicle manufacturing tolerances. The dynamic part is the response of the vehicle in motion to external excitation. The external excitation includes the excitation of the track unevenness, and the unbalanced centrifugal force when passing through the curve. Among them, the unbalanced centrifugal force when passing through the curve makes the car body deflected, which is a steady state quantity. The vibration displacement of the car body caused by the track upset is random and is a random quantity.

\subsubsection{Algorithm}

The UIC boundary standard mainly involves two regulations, UIC505-1 [25] and UIC505-4 [26], which are quite complicated and cumbersome to calculate, and their theoretical principles can be applied to all kinds of rail vehicles. This section will analyze the calculation method of vehicle lateral offset in UIC505 standard limits in detail.

According to the two regulations UIC505-1 and UIC505-4, the basic principle is to calculate the relevant offset in the calculation of vehicle limits and equipment limits of metro trains. We use $D$ to represent the lateral offset of the vehicle, and we can obtain: Transverse drift $(D)$ is composed of the Geometric Offset at Curve $\left(d_{g}\right)$, Wheel-rail clearance, Lateral offset between axle and bogie ( $q$ ), Offset between bogie and car body $(w)$, Quasi-static offset $(z)$, Special items $(X)$.

\subsubsection{Geometric Offset Calculation}

The vehicle will have a geometric offset when crossing a curve, where $d_{g i}$ represents the lateral offset of the vehicle body within the bogie center pin; $d_{g a}$ represents the lateral offset of the vehicle body beyond the bogie center pin; $R$ stands for line curve radius; $a$ is the vehicle fixed distance; $n_{i}$ indicates the distance from the selected section to the center pin of the power bogie (the selected section is between the two axles or the center pin of the bogie); $n_{a}$ indicates the distance from the selected section to the center pin of the power bogie (the selected section is outside the center pin of both axes or bogies); $p$ stands for wheelbase. The geometric offset is calculated as follows $[25,26]$.

$$
\begin{aligned}
& d_{g i}=\frac{1}{2 R}\left(a n_{i}-n_{i}^{2}+\frac{p^{2}}{4}\right) \\
& d_{g a}=\frac{1}{2 R}\left(a n_{a}+n_{a}^{2}-\frac{p^{2}}{4}\right)
\end{aligned}
$$

\subsubsection{Calculation of the Quasi-Static Offset ' $z$ '}

For a suspended body located at a height $h$, we denote the vehicle elasticity coefficient by $s ; \eta_{0}$ denotes the maximum angle of tilting the car body on flat ground; $h$ denotes the height above the running surface; $h_{c}$ denotes the height of the lateral swing center of the car body from the running surface. The quasi-static offset is calculated by the following formula $[25,26]$ :

$$
z=\left[\frac{s}{30}+\tan \left[\eta_{0}-1^{\circ}\right]_{>0}\right]\left|h-h_{c}\right|+\left[\frac{s}{30}\left|h-h_{c}\right|-0.04[h-0.5]_{>0}\right]_{>0}
$$

Special cases: 
Under the conditions of $h>h_{c} \geq 0.5 \mathrm{~m}, s \leq 0.4, \eta_{0} \leq 1^{\circ}$,

$$
z=\frac{s}{30}\left(h-h_{c}\right)
$$

Under the conditions of $h<0.5 \mathrm{~m}, \eta_{0} \leq 1^{\circ}, h_{c}$ and $s$ of whatever value,

$$
z=\frac{4 s}{30}\left|h_{c}-h\right|
$$

Under the condition of $h=h_{c}$ :

$$
z=0
$$

\subsubsection{Calculation of Special Terms $X_{i}, X_{a}$}

For vehicles with a large wheelbase or a large overhang, some of the formulas for calculating the lateral offset are modified to determine whether the outer dimensions of the limits are met when passing curves with radii between $150 \mathrm{~m}$ and $250 \mathrm{~m}[25,26]$.

$X_{i}$ (additional reduction outside the center pin of the extension vehicle) is calculated into the formula only if $\frac{a^{2}+P^{2}}{4}>100$;

$X_{P}$ (additional reduction outside the center pin of the extension vehicle) is only calculated into the formula when $a \cdot n_{a}+a_{n}^{2}-\frac{p^{2}}{4}>120$.

\subsubsection{Lateral Offset of Vehicle through Curved Section}

In this paper, based on the UIC505 limit standard, according to the calculation method in Sections 3.3.1-3.3.4, the effects of Geometric Offset at Curve $\left(d_{g}\right)$, Wheel-rail clearance, Lateral offset between axle and bogie ( $q$ ), Offset between bogie and car body $(w)$, Quasistatic offset $(z)$, and Special term $(X)$ factors under different curve radii are considered. The lateral offset $(D)$ of a train (B-type car) passing through a horizontal curve section with different radii on the actual operating line (Continuous Welded Rail) of Nanchang Metro Line 4 is calculated, and the results are shown in Figure 7.

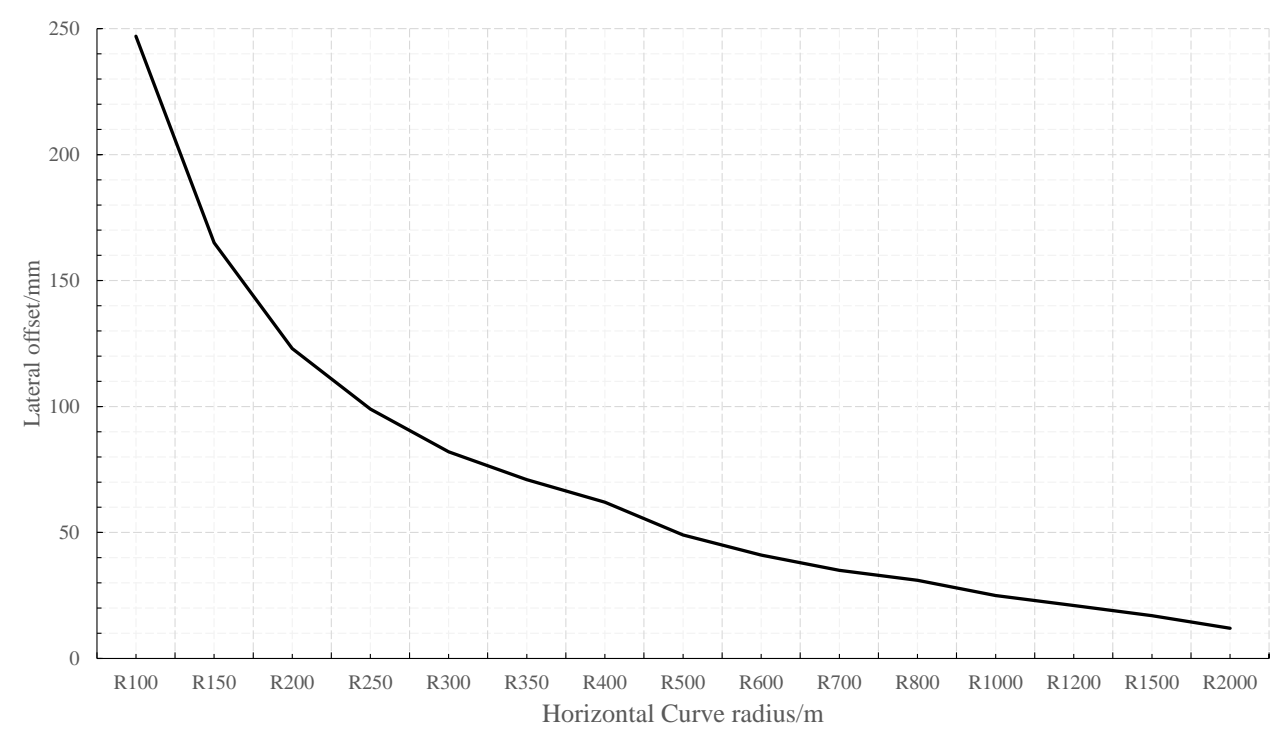

Figure 7. Nanchang Metro Line 4 Train Lateral Offset.

\section{Proposed Method}

\subsection{Algorithm Analysis Steps}

When the subway vehicle passes through the horizontal curve, a certain angle will be generated between the front and rear vehicles, and the size of the angle is greatly related to the safety of the vehicle through the horizontal curve $[27,28]$. Using geometric analysis and 
geometric graphing method, the geometric position and hook swing angle of the vehicle when passing the curve can be calculated.

After completing the design of the line and vehicle characteristics parameters, and selecting the appropriate hook type, the curve linkage performance of the vehicle can be determined. It also determines the maximum number of hook swing angle that the vehicle can achieve when passing the curve, and takes the hook state at this moment as the limit working condition under the corresponding line conditions. At this time, the hook slowing device will unhook, so that the two vehicles are free to approach, to simulate the working scenario of the curve rescue linkage. By calculating and analyzing the trajectory motion characteristics of trains on fixed circular curves, straight line into curves and S-curves to verify whether the hooks can be automatically linked, thus obtaining the actual linkage of hooks on horizontal curves.

The mechanical structure of the coupler head allows a certain horizontal and vertical position difference when the vehicle is automatically connected, i.e., the two hook heads that are being connected theoretically allow automatic connection under working conditions with a relative position difference. After the on-site vehicle connection test, to ensure that the coupler can be safely connected on the curve, it must be ensured that the projection of the apex of the coupler convex cone on the coupler connecting surface is within the connectable area. Figure 8 shows the hooking range of the vehicle [29] .

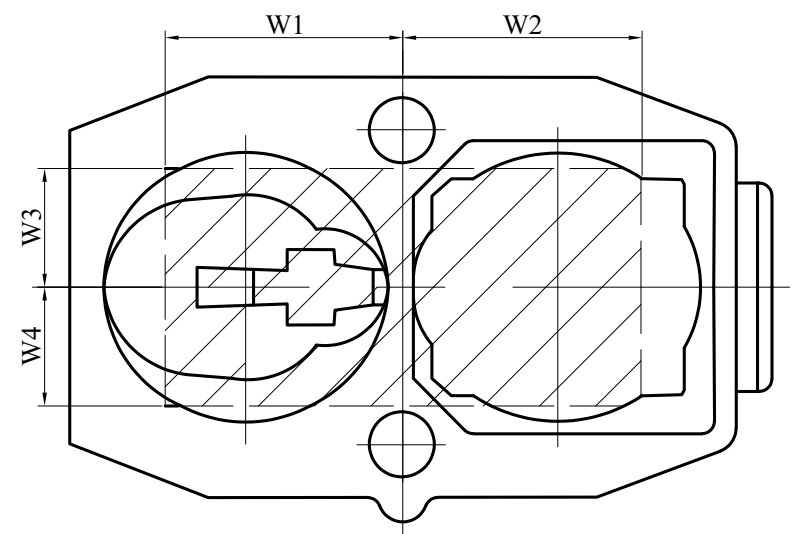

Figure 8. The range of coupler.

Secondly, determine whether the hook body and the line connecting the center of rotation of the hook can form a triangle, Figure 9 shows the schematic diagram of the coupler successful connection, Figure 10 shows the schematic diagram of the coupler failure connection [30].

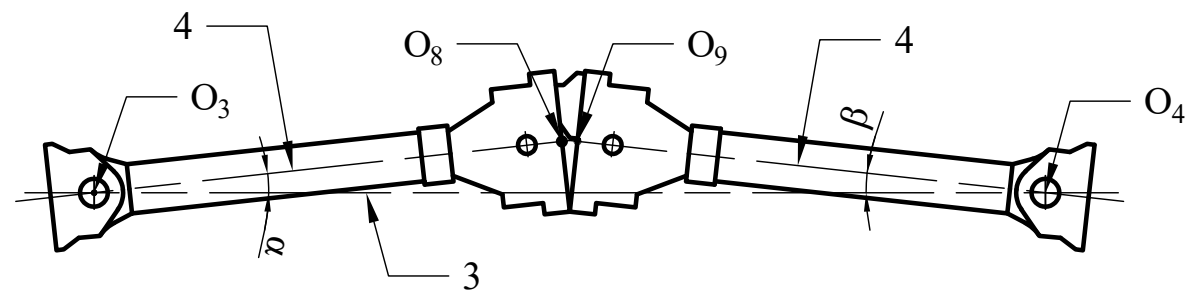

Figure 9. Successful coupling of the coupler. 


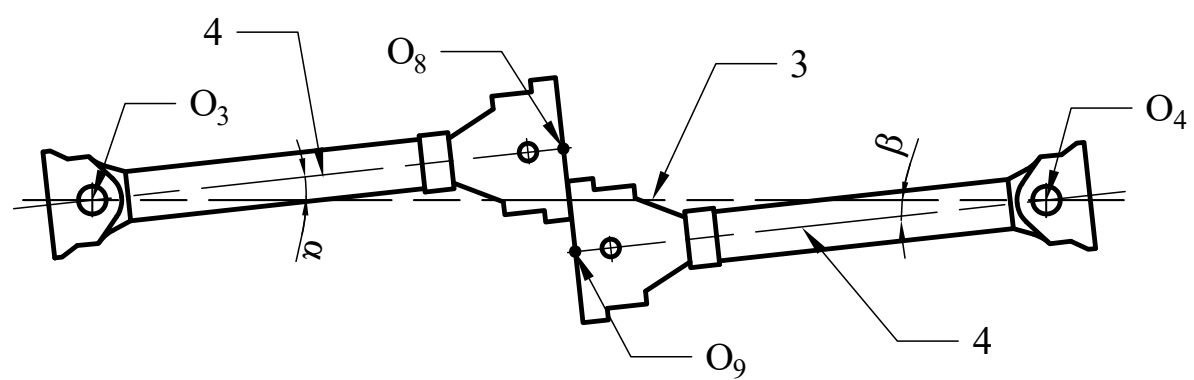

Figure 10. Failure coupling of the coupler.

To simplify the calculation, we use Figure 11 to geometrically simplify the top view of the two vehicle hooks during their connection. In the schematic diagram, the two semi-elliptic curves at point $O_{14}$ and point $O_{15}$ are the simplified graphs of the hook convex cones, and both points are located at the centers of the semi-elliptic curves, which are the vertices of the hook convex cones. The lengths of $O_{10}$ and $O_{11}$ from $O_{8}$ are $W_{1}$ and $W_{2}$, respectively; the lengths of $O_{12}$ and $O_{13}$ from $O_{9}$ are $W_{2}$ and $W_{1}$, respectively. Where the geometric meanings of $W_{1}$ and $W_{2}$ are the same as those of $W_{1}$ and $W_{2}$ in Figure 8.

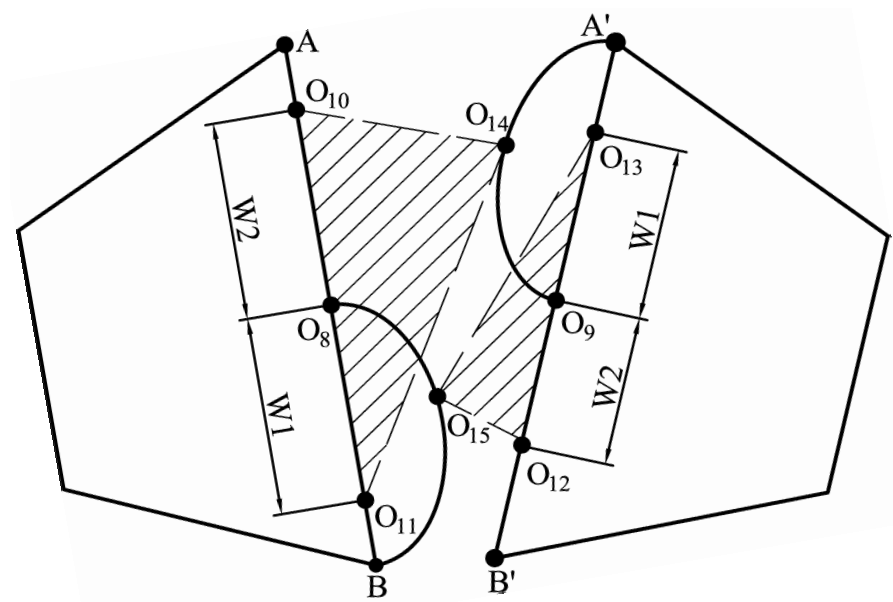

Figure 11. Coupler characteristics triangle.

Finally, as shown in Figure 11, it is necessary to ensure that the orthographic projections of $\mathrm{O}_{14}$ and $\mathrm{O}_{15}$ can fall within line segment $\mathrm{O}_{10} \mathrm{O}_{11}$ and line segment $\mathrm{O}_{12} \mathrm{O}_{13}$, respectively, if the hook can be safely attached on the curve. In other words, it should be ensured that the six angles in the characteristic triangles $\triangle O_{10} O_{11} O_{14}$ and $\triangle O_{12} O_{13} O_{15}$ of the coupler in Figure 10 are all acute angles.

In summary, we propose a calculation method to determine whether the hook can realize the curve rescue linkage, and Algorithm step flow is shown in Figure 12.

\subsection{Determination Method of the Coupler Swing Angle under Horizontal Curve}

Take the example of circular curve working condition, the calculation method of the coupler's swing angle [31,32], Figure 13 shows the calculation diagram of the coupler swing angle calculation method.

In the schematic diagram, $R_{1}$ is the radius of the curved line; $y$ is the maximum offset of the vehicle; $\mathrm{O}_{1}, \mathrm{O}_{2}$ is the rear position car bogie center pin; $\mathrm{O}_{5}, \mathrm{O}_{6}$ is the front position car bogie center pin; $Y_{1}$ is the lateral offset of the center pin $O_{1} ; Y_{2}$ is the lateral offset of center pin $\mathrm{O}_{2} ; Y_{3}$ is the lateral offset of center pin $\mathrm{O}_{5} ; Y_{4}$ is the lateral offset of center pin $\mathrm{O}_{6}$; $\mathrm{O}_{3}$ is the center of rotation of the rear position vehicle; $\mathrm{O}_{4}$ is the center of rotation of the front position vehicle; $L_{2}$ is the rear vehicle bogie wheelbase; $L_{3}$ is the front vehicle bogie wheelbase; $L_{b}$ is the length of the attached hook between vehicles. 


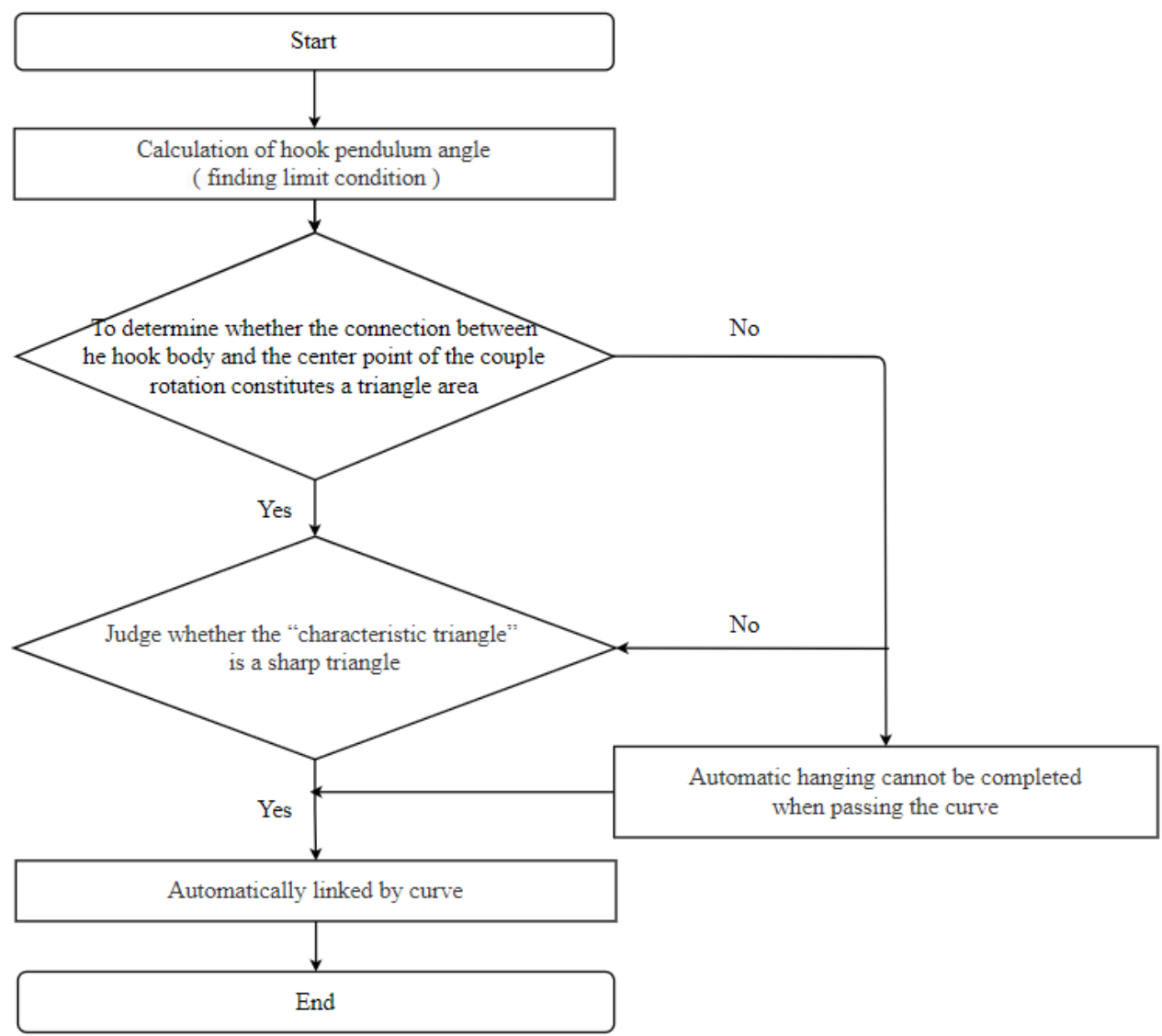

Figure 12. Algorithm step flow chart.

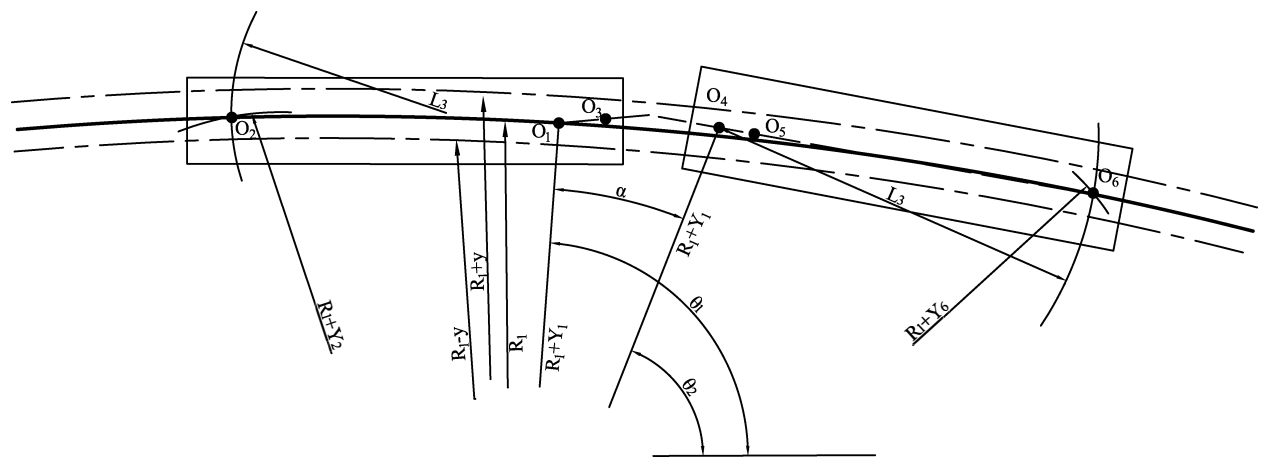

Figure 13. Calculation chart of vehicle for circular curve working condition.

The basic procedure of the calculation is as follows:

Step 1: Establish a coordinate system $O X Y$ with the center of the curve as the origin. The 2-position vehicle's 1-position center pin $O_{1}$ is laterally offset by $Y_{1}$ relative to the center line. The 2-position center pin $\mathrm{O}_{2}$ is laterally offset by $Y_{2}$ relative to the center line. The coordinates of $O_{1}$ are assumed to be $\left(x_{1}, y_{1}\right), \theta_{1}$ denotes the angle between the $O O_{1}$ line and the horizontal coordinate.

Step 2: Take $O_{1}$ as the center, and make a circle with the vehicle distance $L_{3}$ as the radius. Take the center $O(0,0)$ of the center line as the center of another circle, with radius $R+Y_{1}$. Solve the equations of these two circles simultaneously, then obtain the coordinates $\left(x_{2}, y_{2}\right)$ of the intersection point $\mathrm{O}_{2}$ of the two circles.

Step 3: Connect $\mathrm{O}_{1}$ and $\mathrm{O}_{2}$, as the center line of the vehicle body. If 1-position coupler rotation center $\mathrm{O}_{3}$ is located on the extension line of straight line $\mathrm{O}_{1} \mathrm{O}_{2}$, and the distance 
from the center pin $O_{1}$ is $L_{3}$, then the coordinates $\left(x_{3}, y_{3}\right)$ of the rotation center $O_{3}$ of a coupler are:

$$
\begin{aligned}
& x_{3}=\left(x_{1}-x_{2}\right) \frac{L_{2}}{L_{3}}+x_{1} \\
& y_{3}=\left(y_{1}-y_{2}\right) \frac{L_{2}}{L_{3}}+y_{1}
\end{aligned}
$$

Step 4: The 1-position vehicle's 2-position center pin $O_{5}$ is laterally offset by $Y_{3}$ relative to the center line. The 1-position center pin $O_{6}$ is laterally offset by $Y_{4}$ relative to the center line. At this time, the coordinate position of $\mathrm{O}_{5}$ needs to be estimated based on the angle $\alpha$ between $\mathrm{OO}_{5}$ and $\mathrm{OO}_{1}$; assume that the coordinates of $\mathrm{O}_{5}$ are $\left(x_{5}, y_{5}\right), \theta_{2}$ denotes the angle between the $\mathrm{OO}_{5}$ line and the horizontal coordinate.

Step 5: Make a circle with center $\mathrm{O}_{5}$, and the vehicle distance $L_{3}$ as the radius. Take the center $\mathrm{O}(0,0)$ of the center line as the center of another circle with radius $\mathrm{R}+Y_{4}$. Solve the equations of these two circles simultaneously. The coordinates of the intersection point $\mathrm{O}_{6}$ of the two circles $\left(x_{6}, y_{6}\right)$ can be obtained.

Step 6: Connect $\mathrm{O}_{5}$ and $\mathrm{O}_{6}$ to obtain the center line of the vehicle body. The car body extends along this line in an axial symmetry distribution. The 2-position coupler rotation center $\mathrm{O}_{4}$ is located on the extension of straight line $\mathrm{O}_{5} \mathrm{O}_{6}$, and the distance from the center pin $\mathrm{O}_{5}$ is $\mathrm{L}_{3}$. Then, the coordinates $\left(x_{4}, y_{4}\right)$ of the rotation center $\mathrm{O}_{4}$ of the 2-position coupler is:

$$
\begin{aligned}
& x_{4}=\left(x_{5}-x_{6}\right) \frac{L_{2}}{L_{3}}+x_{5} \\
& y_{4}=\left(y_{5}-y_{6}\right) \frac{L_{2}}{L_{3}}+y_{5}
\end{aligned}
$$

Step 7: Determine the position of the 1-position vehicle by determining the length of the coupler. First, according to the front and rear vehicle coupler rotation centers $\mathrm{O}_{3}\left(x_{3}, y_{3}\right)$ and $\mathrm{O}_{4}\left(x_{4}, y_{4}\right)$, calculate the coupler length $L_{b}$, Figure 14 shows the geometric schematic of the vehicle hook length calculation.

$$
L_{b}=\sqrt{\left(x_{4}-x_{3}\right)^{2}+\left(y_{4}-y_{3}\right)^{2}}
$$

Step 8:As shown in Figure 14, determine the threshold as $\phi$, if $\left|L_{b}-L_{0}\right| \leqslant \phi$, continue to Step 9 to calculate the swing angle of the coupler. If $\left|L_{b}-L_{0}\right|>\phi$, modify $\theta_{2}$, and repeat Steps 4 to 7 until the requirements are met. Continue to Step 9 to calculate the coupler swing angle.

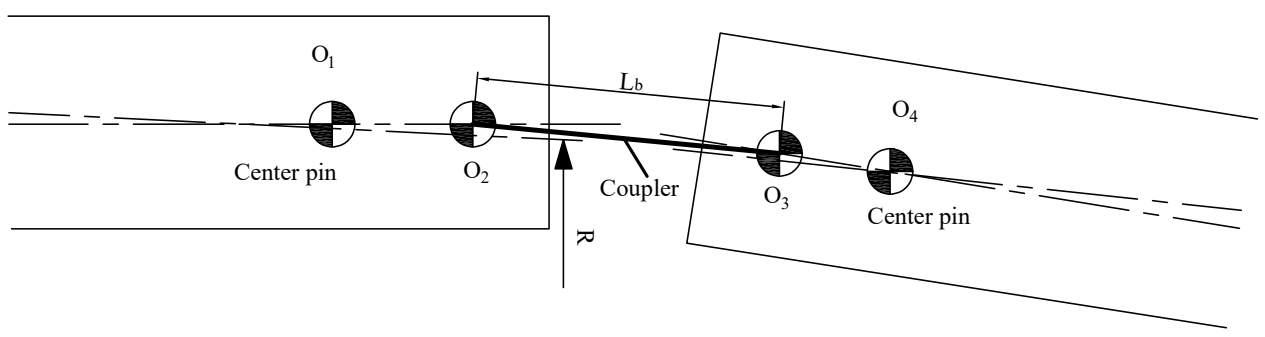

Figure 14. Vehicle coupler length calculation chart.

Step 9: Calculate the swing angle of the coupler. Calculate the angles between the straight lines $\mathrm{O}_{1} \mathrm{O}_{2}$ and $\mathrm{O}_{3} \mathrm{O}_{4}$, and the straight lines $\mathrm{O}_{5} \mathrm{O}_{6}$ and $\mathrm{O}_{3} \mathrm{O}_{4}$.

\subsection{Determination Method of Hook Position under Horizontal Curve}

Take the example of circular curve working condition, the two couplers are successfully connected under the guidance of the guide rod after the above conditions are met. Figure 15 shows the geometric diagram of the calculation method for coupler connection. 


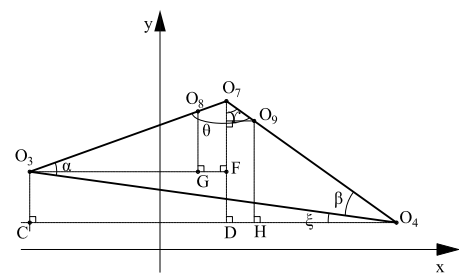

(a)

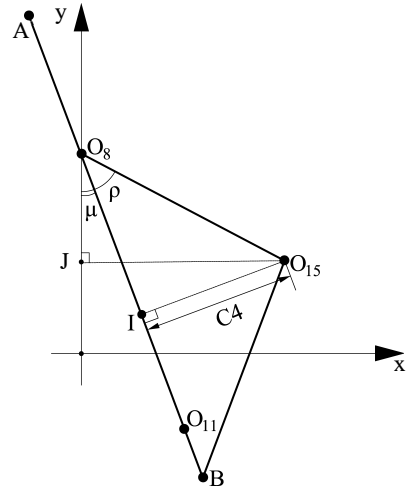

(b)

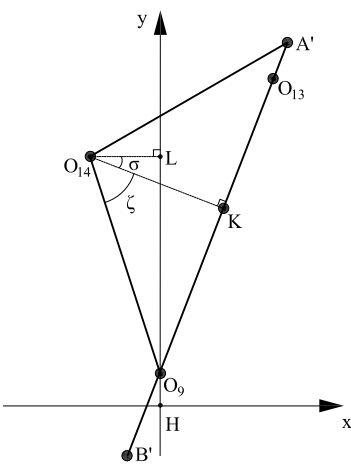

(c)

Figure 15. Geometric calculation diagram of coupler connection. (a) Geometric calculation of the coordinates of the vertices associated with the characteristic triangle of the vehicle hook 1 ; (b) Geometric calculation of the coordinates of the vertices associated with the characteristic triangle of the vehicle hook 2; (c) Geometric calculation of the size of the interior angles of the characteristic triangle of the vehicle hook.

As shown in Figure 15, point $\mathrm{O}_{3}$ is the center of rotation of the hook of the No.1 vehicle and point $\mathrm{O}_{4}$ is the center of rotation of the hook of the No.2 vehicle; $\mathrm{O}_{8}$ and $\mathrm{O}_{9}$ are the center points of the ends of the hooks of the two carriage hooks; $O_{7}$ is the intersection of the centerline of the hook bodies of the two hooks; the angles $\alpha$ and $\beta$ are the front and rear hook swing angles calculated in Section 3.3.1; the points $O_{10} \ldots O_{15}$ represent the same geometric meaning as that represented by $O_{10} \ldots O_{15}$ in Figure 11.

Step 1: As shown in Figure 15a, establish a coordinate system $O X Y$ with the center of the curve as the origin. From the vehicle body pose calculation method, the coordinates of $\mathrm{O}_{3}$ are $\left(x_{3}, y_{3}\right)$ and the coordinates of $O_{4}$ are $\left(x_{4}, y_{4}\right)$, and the left and right swing angles are $\alpha$ and $\beta$ after the coupler is connected.

Step 2: For connection point $\mathrm{O}_{3}$ and point $\mathrm{O}_{4}$, make an extension line along the center line of the coupler through two points $\mathrm{O}_{3}$ and $\mathrm{O}_{4}$. If the two straight lines intersect at a point $O_{7}$, so that $\mathrm{O}_{3}, \mathrm{O}_{4}$ and $\mathrm{O}_{7}$ can form a triangle, then the next step can be calculated. Otherwise, the coupler will not be normally automatically connected and manual assistance is required.

Step 3: Make a vertical line perpendicular to the $X$-axis through $O_{3}$, and make a straight line parallel to the $X$ - axis through $O_{4}$. The two lines intersect at a point $C$, let $\angle \mathrm{O}_{3} \mathrm{O}_{4} \mathrm{C}$ be $\xi$. Cross $\mathrm{O}_{7}$ to make a perpendicular line to cross $\mathrm{O}_{4} \mathrm{C}$ at point $\mathrm{D}$, let $\angle D O_{7} \mathrm{O}_{4}$ be $\gamma$. Let $\angle \mathrm{O}_{3} \mathrm{O}_{7} \mathrm{O}_{4}$ be $\theta$, then:

$$
\begin{gathered}
\xi=\cos ^{-1} \frac{O_{4} O_{3}}{O_{4} C} \\
\gamma=90^{\circ}-(\delta+\beta) \\
\theta=180^{\circ}-\alpha-\beta
\end{gathered}
$$

The coordinates of $O_{7}\left(x_{7}, y_{7}\right)$ are:

$$
\begin{gathered}
x_{7}=x_{4}-\frac{\sin \alpha \cdot O_{3} O_{4}}{\sin \varepsilon} \cdot \cos \varepsilon \\
y_{7}=y_{4}+\sin \alpha \cdot O_{3} O_{4}
\end{gathered}
$$


Step 4: Make a perpendicular line passing $O_{3}$ to intersect $O_{7} D$ at point $F$, and then make a perpendicular line passing $\mathrm{O}_{8}$ to intersect $\mathrm{O}_{3} \mathrm{~F}$ at point $\mathrm{G}$, then the coordinates of $\mathrm{O}_{8}\left(x_{8}, y_{8}\right)$ are:

$$
\begin{aligned}
& x_{8}=\frac{O_{3} F \cdot L_{4}}{O_{3} O_{7}}-\left|x_{3}\right| \\
& y_{8}=\frac{O_{7} F \cdot L_{4}}{O_{3} O_{7}}+y_{3}
\end{aligned}
$$

In the same way, cross $\mathrm{O}_{9}$ to make a perpendicular line to intersect $\mathrm{O}_{4} \mathrm{C}$ at point $\mathrm{H}$, then the coordinates of $O_{9}\left(x_{9}, y_{9}\right)$ are:

$$
\begin{aligned}
& x_{9}=x_{4}-\frac{O_{4} D \cdot L_{4}}{O_{4} O_{7}} \\
& y_{9}=\frac{O_{7} F \cdot L_{4}}{O_{4} O_{7}}+y_{4}
\end{aligned}
$$

Step 5: As shown in Figure 15, by determining the slope of the straight line $A B$ and the straight line $A^{\prime} B^{\prime}$, the coordinates $\left(x_{10}, y_{10}\right)$ of point $O_{10}$ and the coordinates $\left(x_{11}, y_{11}\right)$ of $O_{11}$ are obtained as:

$$
\begin{gathered}
K_{A B}=\frac{x_{3}-x_{7}}{y_{7}-y_{3}} \\
K_{A^{\prime} B^{\prime}}=\frac{x_{4}-x_{7}}{y_{7}-y_{4}} \\
x_{10}=x_{8}-W_{1} \cdot \cos \left(\tan ^{-1} K_{A B}\right) \\
y_{10}=y_{8}+W_{1} \cdot \sin \left(\tan ^{-1} K_{A B}\right) \\
x_{11}=x_{8}+W_{1} \cdot \cos \left(\tan ^{-1} K_{A B}\right) \\
y_{11}=y_{8}-W_{1} \cdot \sin \left(\tan ^{-1} K_{A B}\right)
\end{gathered}
$$

In the same way, as shown in Figure 15c, it can be concluded that the coordinates of point $O_{12}\left(x_{12}, y_{12}\right)$ and the coordinates $\left(x_{13}, y_{13}\right)$ of $O_{13}$ are:

$$
\begin{aligned}
& x_{12}=x_{9}-W_{1} \cdot \cos \left(\tan ^{-1} K_{A^{\prime} B^{\prime}}\right) \\
& y_{12}=y_{9}-W_{1} \cdot \sin \left(\tan ^{-1} K_{A^{\prime} B^{\prime}}\right) \\
& x_{13}=x_{9}+W_{1} \cdot \cos \left(\tan ^{-1} K_{A^{\prime} B^{\prime}}\right) \\
& y_{13}=y_{9}+W_{1} \cdot \sin \left(\tan ^{-1} K_{A^{\prime} B^{\prime}}\right)
\end{aligned}
$$

Step 6: As shown in Figure 15b, cross point $O_{15}$ to make a perpendicular line and cross AB at point $I$. Cross point $O_{15}$ to make a perpendicular line to cross $O_{8} G$ at point $J$, let $\angle J O_{8} I$ be $\mu$, let $\angle I O_{8} O_{15}$ be $\rho$, then the coordinates of point $O_{15}\left(x_{15}, y_{15}\right)$ are:

$$
\begin{aligned}
& x_{15}=x_{8}+\sqrt{C_{4}^{2}+\left(\frac{C_{2}}{4}\right)^{2}} \cdot \sin \left(\tan ^{-1} \frac{4 C_{4}}{C_{2}}+\tan ^{-1} \frac{1}{K_{A B}}\right) \\
& y_{15}=y_{8}-\sqrt{C_{4}^{2}+\left(\frac{C_{2}}{4}\right)^{2}} \cdot \cos \left(\tan ^{-1} \frac{4 C_{4}}{C_{2}}+\tan ^{-1} \frac{1}{K_{A B}}\right)
\end{aligned}
$$

In the same way, cross $O_{14}$ to make a perpendicular line $A^{\prime} B^{\prime}$ at point $K$, and cross point $O_{14}$ to make a perpendicular line to cross $O_{9} H$ at point $L$. Let $\angle K O_{14} L$ be $\sigma$, and let $\angle K O_{14} O_{9}$ be $\zeta$, then the coordinates $\left(x_{14}, y_{14}\right)$ of point $O_{14}$ are:

$$
x_{14}=x_{9}-\sqrt{C_{4}^{2}+\left(\frac{C_{2}}{4}\right)^{2}} \cdot \cos \left(\tan ^{-1} \frac{4 C_{4}}{C_{2}}+\tan ^{-1} \frac{1}{K_{A^{\prime} B^{\prime}}}\right)
$$




$$
y_{15}=y_{9}+\sqrt{C_{4}^{2}+\left(\frac{C_{2}}{4}\right)^{2}} \cdot \sin \left(\tan ^{-1} \frac{4 C_{4}}{C_{2}}+\tan ^{-1} \frac{1}{K_{A^{\prime} B^{\prime}}}\right)
$$

Step 7: As shown in Figure 11, when the coordinates of each point of $\triangle O_{10} O_{11} O_{14}$ and $\triangle \mathrm{O}_{12} \mathrm{O}_{13} \mathrm{O}_{15}$ are known, the cosine value of each angle in the two triangles can be obtained:

$$
\begin{aligned}
& \cos \angle O_{10} O_{11} O_{14}=\frac{O_{10} O_{11}{ }^{2}+O_{11} O_{14}{ }^{2}-O_{10} O_{14}{ }^{2}}{2 \cdot O_{10} O_{11} \cdot O_{11} O_{14}} \\
& \cos \angle O_{11} O_{10} O_{14}=\frac{O_{10} O_{11}{ }^{2}+O_{10} O_{14}{ }^{2}-O_{11} O_{14}{ }^{2}}{2 \cdot O_{10} O_{11} \cdot O_{10} O_{14}} \\
& \cos \angle O_{10} O_{14} O_{11}=\frac{O_{10} O_{14}{ }^{2}+O_{11} O_{14}{ }^{2}-O_{10} O_{11}{ }^{2}}{2 \cdot O_{10} O_{14} \cdot O_{11} O_{14}} \\
& \cos \angle O_{12} O_{13} O_{15}=\frac{O_{13} O_{15}{ }^{2}+O_{13} O_{12}{ }^{2}-O_{15} O_{12}{ }^{2}}{2 \cdot O_{13} O_{15} \cdot O_{13} O_{12}} \\
& \cos \angle O_{12} O_{15} O_{13}=\frac{O_{13} O_{15}{ }^{2}+O_{15} O_{12}{ }^{2}-O_{13} O_{12}{ }^{2}}{2 \cdot O_{13} O_{15} \cdot O_{15} O_{12}} \\
& \cos \angle O_{15} O_{12} O_{13}=\frac{O_{13} O_{12}{ }^{2}+O_{15} O_{12}{ }^{2}-O_{13} O_{15}{ }^{2}}{2 \cdot O_{13} O_{12} \cdot O_{15} O_{12}}
\end{aligned}
$$

Step 8: If the cosine values of the six corners are all greater than 0 , the six corners are all acute angles, which meets the requirements for automatic coupling of couplers. If the cosine values of the six angles are not all greater than 0 , the six angles are not all acute angles, which cannot meet the requirements for automatic coupling of the coupler.

\section{Analysis of Coupler Curve Connection under Different Working Conditions}

This paper takes the Nanchang Metro Line 4 train, which will be opened for operation in 2021, as the research object. Based on the line level and longitudinal section data map of Nanchang Metro Line 4 Phase I Project, the actual line working conditions through which the metro trains will be operated in the future are investigated and analyzed to obtain the corresponding limit working conditions under different curve-line working conditions. The investigation results show that: at $445.777 \mathrm{~km} \sim 759.731 \mathrm{~km}$ on the left side of the main line, the radius of the circular curve reaches the minimum, and the radius of the curve is $330 \mathrm{~m}$; at $444.175 \mathrm{~km} \sim 754.205 \mathrm{~km}$ on the right side of the main line, the curve radius reaches the minimum when the straight line enters the curve, and the curve radius is $325 \mathrm{~m}$; in the Wangcheng vehicle section there is S-curve, its front curve radius of $255 \mathrm{~m}$ after the curve radius of $250 \mathrm{~m}$, the straight line between the two curves is $38.853 \mathrm{~m}$.

\subsection{Circular Curve Connection}

\subsubsection{Determination of Limiting Working Conditions}

Based on the algorithm presented in Section 4, the calculation of the vehicle position and the swing angle of the hook in a circular curve working condition can determine the limit working condition of the train when the rescue linkage is performed on a circular curve.

When the vehicle passes the left line of the main line at $445.777 \mathrm{~km} \sim 759.731 \mathrm{~km}$, the radius of the circle curve reaches the minimum, and the radius of the curve is $330 \mathrm{~m}$. It is calculated that the lateral offset of the vehicle is $75 \mathrm{~mm}$. At this time, the maximum swing angle of the hook at this time appears when the front and rear center pins of the two vehicles are intersect with each other. Table 2 is the maximum values of the coupler swing angle of the vehicle in the circular curve working condition. The maximum coupler swing angle for No.2 coupler of No.1 vehicle is $8.4963^{\circ}$, the maximum coupler swing angle for No.1 coupler of No.2 is $7.7047^{\circ}$. The position where the maximum swing angle of the coupler appears is the limit position of the vehicle under the condition of the line. 
Table 2. Maximum swing angle of the coupler under circular curve conditions.

\begin{tabular}{ccccccc}
\hline & \multicolumn{2}{c}{ Coupler Swing Angle $\left(^{\circ}\right)$} & \multicolumn{4}{c}{ Center Pin Lateral Offset (mm) } \\
\cline { 2 - 7 } S/N & No.1 Vehicle & No.2 Vehicle & $\begin{array}{c}\text { No.1 Coupler of } \\
\text { No.1 Vehicle }\end{array}$ & $\begin{array}{c}\text { No.2 Coupler of } \\
\text { No.1 Vehicle }\end{array}$ & $\begin{array}{c}\text { No.1 Coupler of } \\
\text { No.2 Vehicle }\end{array}$ & $\begin{array}{c}\text { No.2 Coupler of } \\
\text { No.2 Vehicle }\end{array}$ \\
\hline 1 & 5.0384 & 8.4963 & 75 & -75 & 75 & -75 \\
2 & 3.346 & 7.7047 & -75 & 75 & -75 & 75 \\
\hline
\end{tabular}

\subsubsection{Feasibility Analysis of the Curve Rescue Linkage}

After the limit working condition is determined, the "characteristic triangle" calculation is used to determine whether the end of the hook can fall within the hook attachment area based on the calculation method introduced in Section 4.3. At this point, the hook position diagram is shown in Figure 16.

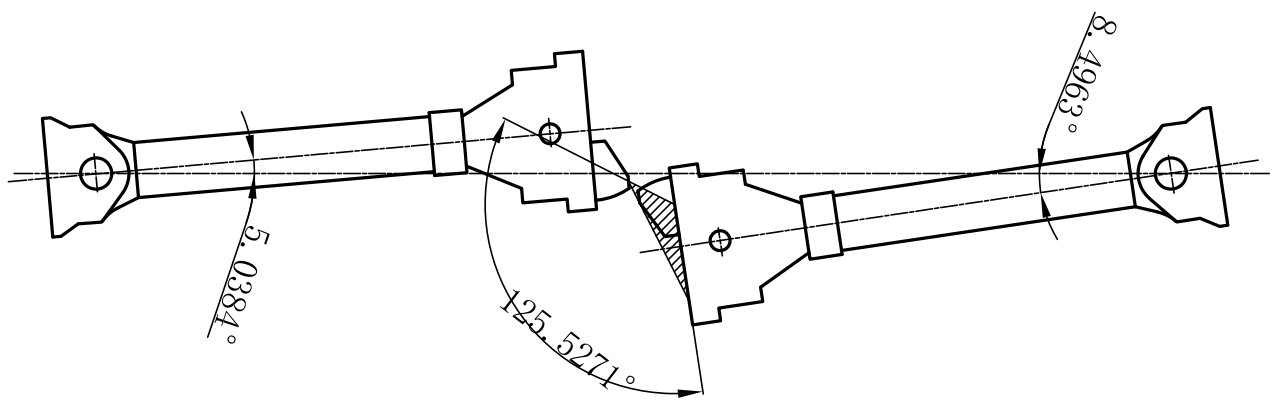

(a) $\mathrm{S} / \mathrm{N} 1$

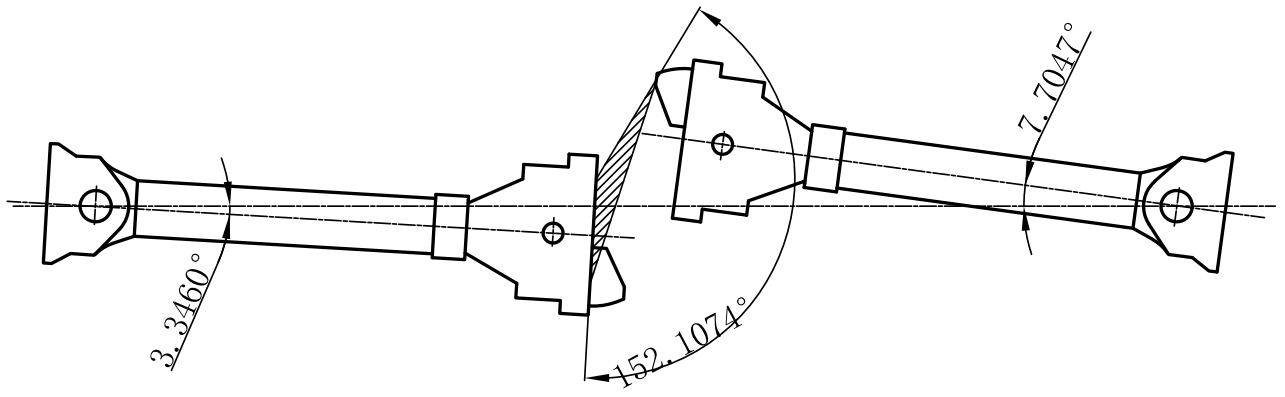

(b) $\mathrm{S} / \mathrm{N} 2$

Figure 16. The position of the hook under the circular curve working condition. (a) Hook position state at the maximum swing angle of coupler No.2 of vehicle No.1 under circular curve working condition; (b) Hook position state at the maximum swing angle of coupler No.1 of vehicle No.2 under circular curve working condition.

From this figure, it can be easily concluded that the characteristic triangle between the two vehicle hooks is an obtuse triangle. At this time, the convex cone of the front vehicle cannot be inserted into the concave cone of the rear vehicle, do not meet the requirements of safe automatic connection, i.e., the couplers cannot complete automatic connection on the R330m fixed circular curve connection, and manual auxiliary connection is required.

\subsection{Curve-Line Working Condition}

When the vehicle enters the curve, there are three different states: the 2-position center pin of the 1-position vehicle, and the 1-position center pin of the 2-position vehicle are located on the straight horizontal area; the 2-position center pin of the 1-position vehicle is located on the curve part of the line, and the 1-position center pin of the 2-position vehicle is located on the straight horizontal area; the 2-position center pin of the 1-position vehicle and the 1-position center pin of the 2-position vehicle are both located on the curved part 
of the line. The analysis algorithm to determine whether a rescue streak can be performed is the same as the determination method on the circle curve.

\subsubsection{Determination of Limiting Working Conditions}

When the vehicle passes the right line of the main line at $444.175 \mathrm{~km}-754.205 \mathrm{~km}$, the curve radius reaches the minimum when the straight line enters the curve, and the curve radius is $325 \mathrm{~m}$. Under this working condition, the straight connection point is used as the starting zero point of the line, and the center line of the front and back two coupler connecting noodles is used as the reference line to determine the limit working position of the coupler's swing angle on the entire line [33,34].

It is calculated that the lateral offset of the train is $83.5 \mathrm{~mm}$. At this time, the maximum swing angle of the coupler occurs when the front and rear center pins of the two vehicles are interlaced with each other. Figure 17 shows the change curve of the vehicle coupler's swing angle at different positions when entering the curve. As Table 3 shows, the maximum coupler swing angle for No.2 coupler of No.1 vehicle appears at $1.0 \mathrm{~m}$ into the curve, and the maximum is $17.3957^{\circ}$. The maximum coupler swing angle for No.1 coupler of No.2 vehicle appears at the entry curve $-2.5 \mathrm{~m}$, and the maximum is $15.6451^{\circ}$. The position where the maximum swing angle of the coupler appears is the limit position of the vehicle under the condition of the line.

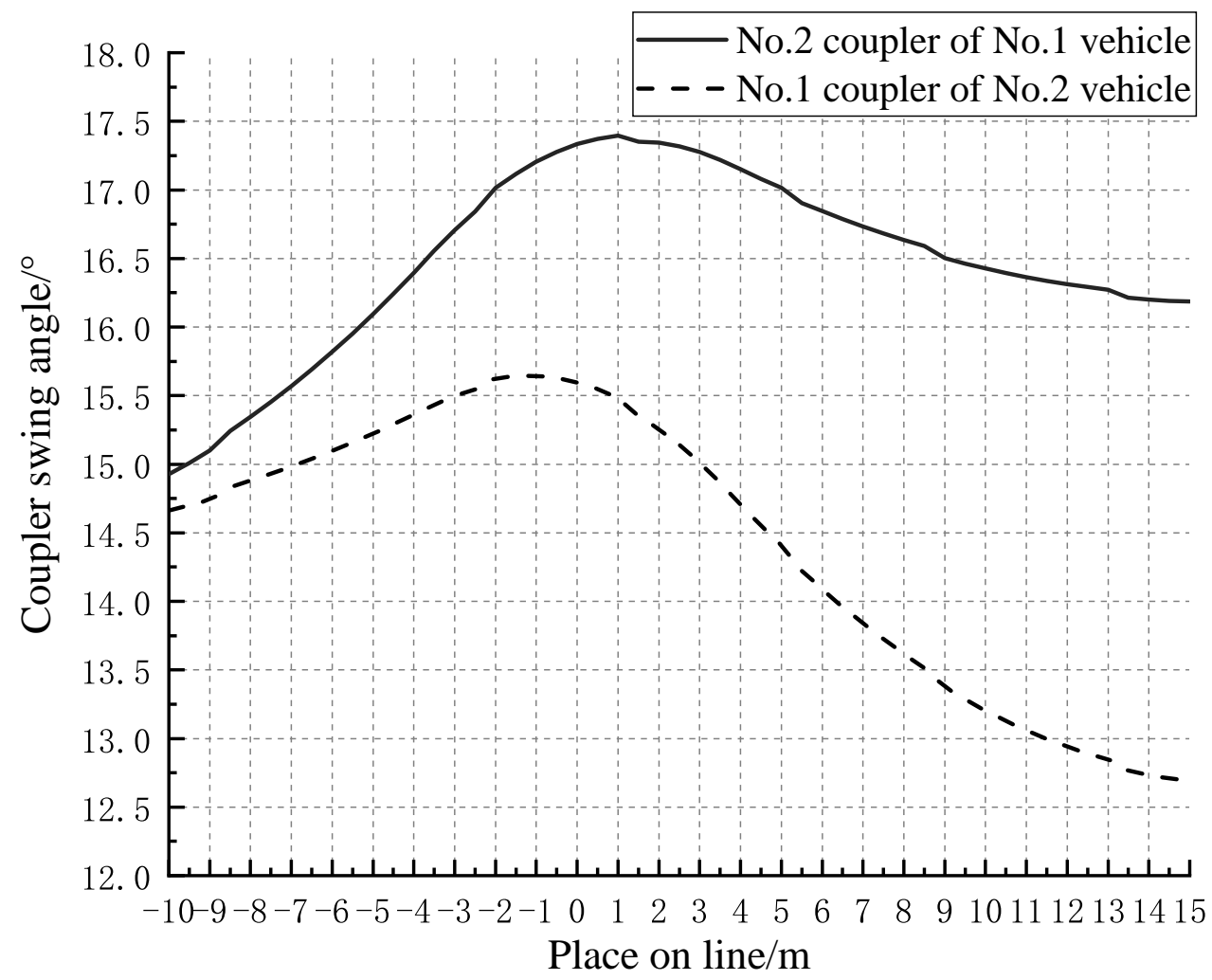

Figure 17. The change curve of the swing angle of the coupler at different positions under curve-line working connection.

Under the curve-line working connection, the maximum swing angle of the coupler appears when No.1 vehicle has just entered the curve and the No.2 vehicle has not yet entered the curve, and the swing angle of the coupler gradually decreases as the No.2 vehicle enters the curve; At the same time, the coupler swing angle of No.2 vehicle is always smaller than the coupler swing angle of No.1 vehicle. Therefore, we should focus on checking the coupler swing angle of No.1 vehicle when it enters the curve, and try to reduce the chance of curve rescue linkage operation at this location to ensure the safety of the train. 
Table 3. The maximum swing angle of the coupler under curve-line working condition.

\begin{tabular}{cccccccc}
\hline & \multicolumn{2}{c}{ Coupler Swing Angle $\left(^{\circ}\right)$} & \multicolumn{2}{c}{ Center Pin Lateral Offset (mm) } \\
\cline { 2 - 7 } S/N & Position & $\begin{array}{c}\text { No.1 } \\
\text { Vehicle }\end{array}$ & $\begin{array}{c}\text { No.2 } \\
\text { Vehicle }\end{array}$ & $\begin{array}{c}\text { No.1 Coupler of } \\
\text { No.1 Vehicle }\end{array}$ & $\begin{array}{c}\text { No.2 Coupler of } \\
\text { No.1 Vehicle }\end{array}$ & $\begin{array}{c}\text { No.1 Coupler of } \\
\text { No.2 Vehicle }\end{array}$ & $\begin{array}{c}\text { No.2 Coupler of } \\
\text { No.2 Vehicle }\end{array}$ \\
\hline 1 & 1.0 & 17.3957 & 15.4818 & -83.5 & 83.5 & -83.5 & 83.5 \\
2 & -2.5 & 17.1185 & 15.6451 & 83.5 & 83.5 & -83.5 & 83.5 \\
\hline
\end{tabular}

\subsubsection{Feasibility Analysis of the Curve Rescue Linkage}

After the limit working condition is determined, the "characteristic triangle" calculation is used to determine whether the end of the hook can fall within the hook attachment area based on the calculation method introduced in Section 4.3. At this point, the hook position diagram is shown in Figure 18.

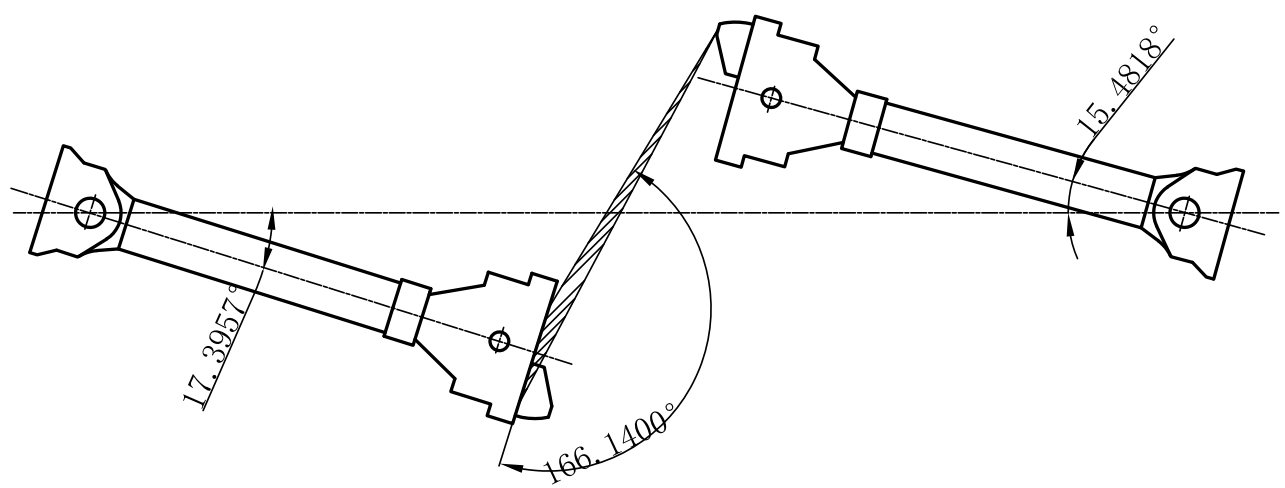

(a) $\mathrm{S} / \mathrm{N} 1$

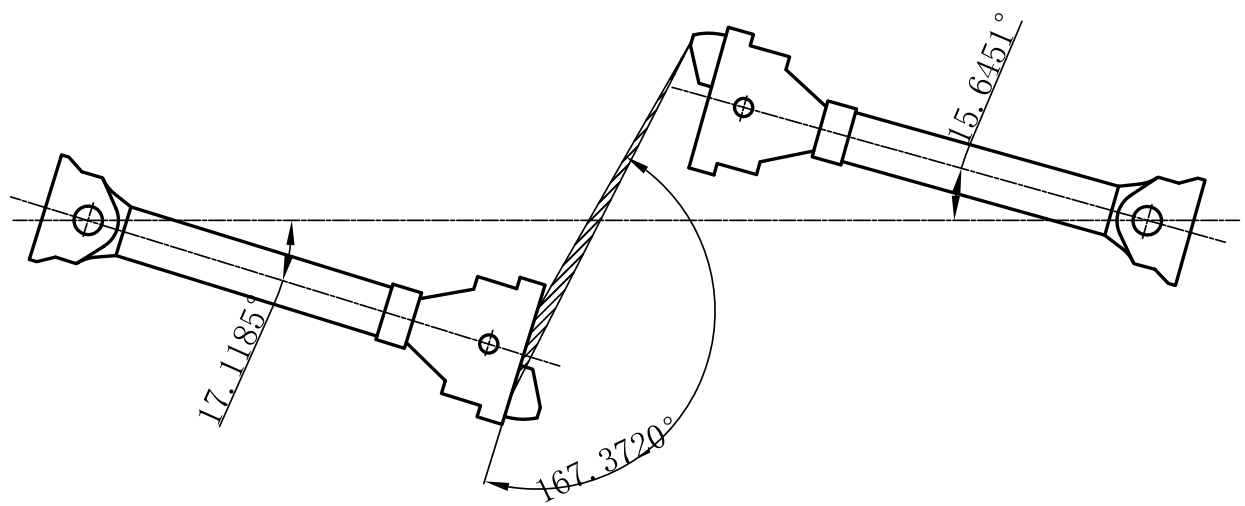

(b) $\mathrm{S} / \mathrm{N} 2$

Figure 18. The position of the hook under the Curve-line working condition. (a) Hook position state at the maximum swing angle of coupler No.2 of vehicle No.1 under circular curve-line working condition; (b) Hook position state at the maximum swing angle of coupler No.1 of vehicle No.2 under circular curve-line working condition.

From this figure, it can be easily concluded that the characteristic triangle between the two vehicle hooks is an obtuse triangle. At this time, the convex cone of the front vehicle cannot be inserted into the concave cone of the rear vehicle, do not meet the requirements of safe automatic connection, i.e., the couplers cannot complete automatic connection on the R325 m straight line into curve connection, and manual auxiliary connection is required. 


\subsection{S-Curve Connection}

When the vehicle is running on the S-curve, there are five different states: the second center pin of the 1-position vehicle and the 1-position center pin of the 2-position vehicle are located in the second half of the S-curve; the 1-position center pin of the 2-position vehicle is located in the second half of the S-curve, and the 2-position center pin of the 1-position vehicle is located in the straight part of the S-curve; the 1-position center pin of the 2-position vehicle and the 2-position center pin of the 1-position vehicle are both located in the straight part of the S-curve; the 1-position center pin of the 2-position vehicle is located in the straight part of the S-curve, and the 2-position center pin of the 1-position vehicle is located in the first half of the S-curve; both the 1-position center pin of the 2-position vehicle and the 2-position center pin of the 1-position vehicle are located in the first half of the S-curve. The analysis algorithm to determine whether a rescue streak can be performed is the same as the determination method on the circle curve.

\subsubsection{Determination of Limiting Working Conditions}

When the vehicle passes through the Wangcheng vehicle section, it will pass through the only S-curve in the line, whose front curve radius is $255 \mathrm{~m}$, the back curve radius is $250 \mathrm{~m}$, and the straight line between the two curves is $38.853 \mathrm{~m}$. Under this condition, the first straight connection point of the S-curve from left to right is used as the starting zero point of the line, and the center line of the front and back two couple connecting noodles is used as the reference line to determine the limit working position of the coupler swing angle on the entire line.

It is calculated that the lateral offset of the train is $99 \mathrm{~mm}$. At this time, the maximum swing angle of the coupler occurs when the front and rear center pins of the two vehicles are interlaced with each other. Figure 19 shows the change curve of the coupler's swing angle in different positions of the vehicle under the S-curve working condition. As Table 4 shows, the maximum coupler swing angle for No.1 coupler of No.2 vehicle appears at $3.818 \mathrm{~m}$ where the center of the coupler is attached into the rear section curve, the maximum value is $10.132^{\circ}$; the maximum coupler swing angle for No.1 coupler of No.2 vehicle appears at the entry curve $4.0 \mathrm{~m}$, the maximum value is $10.146^{\circ}$. This position is the limit position of the vehicle under the condition of the line.

Under the S-curve working condition, the maximum swing angle of the coupler of the No. 2 vehicle appears when the No.1 coupler enters the rear curve and the No.2 vehicle is in the clamped straight line; As the No.2 vehicle also enter the curve section, the maximum swing angle of the No.1 vehicle coupler gradually becomes larger, and the swing angle of the No.2 vehicle hook gradually becomes smaller. Therefore, in the S-curve working condition, we should focus on checking the coupler swing angle when the No.1 vehicle enters the rear curve completely while the No.2 vehicle are still in the clamped straight line, and try to reduce the chance of curve rescue linkage operation at this location to ensure the safety of the train.

Table 4. The maximum swing angle of the coupler under S-curve condition.

\begin{tabular}{cccccccc}
\hline & & \multicolumn{2}{c}{ Coupler Swing Angle $\left(^{\circ}\right)$} & \multicolumn{2}{c}{ Center Pin Lateral Offset (mm) } \\
\cline { 2 - 8 } S/N & Position & $\begin{array}{c}\text { No.1 } \\
\text { Vehicle }\end{array}$ & $\begin{array}{c}\text { No.2 } \\
\text { Vehicle }\end{array}$ & $\begin{array}{c}\text { No.1 Coupler of } \\
\text { No.1 Vehicle }\end{array}$ & $\begin{array}{c}\text { No.2 Coupler of } \\
\text { No.1 Vehicle }\end{array}$ & $\begin{array}{c}\text { No.1 Coupler of } \\
\text { No.2 Vehicle }\end{array}$ & $\begin{array}{c}\text { No.2 Coupler of } \\
\text { No.2 Vehicle }\end{array}$ \\
\hline 1 & 42.671 & 10.132 & 6.975 & -99 & 99 & 99 & -99 \\
2 & 4.0 & 6.886 & 10.146 & -99 & -99 & 99 & 99 \\
\hline
\end{tabular}

\subsubsection{Feasibility Analysis of the Curve Rescue Linkage}

After the limit working condition is determined, the "characteristic triangle" calculation is used to determine whether the end of the hook can fall within the hook attachment area based on the calculation method introduced in Section 4.3. At this point, the hook position diagram is shown in Figure 20. 


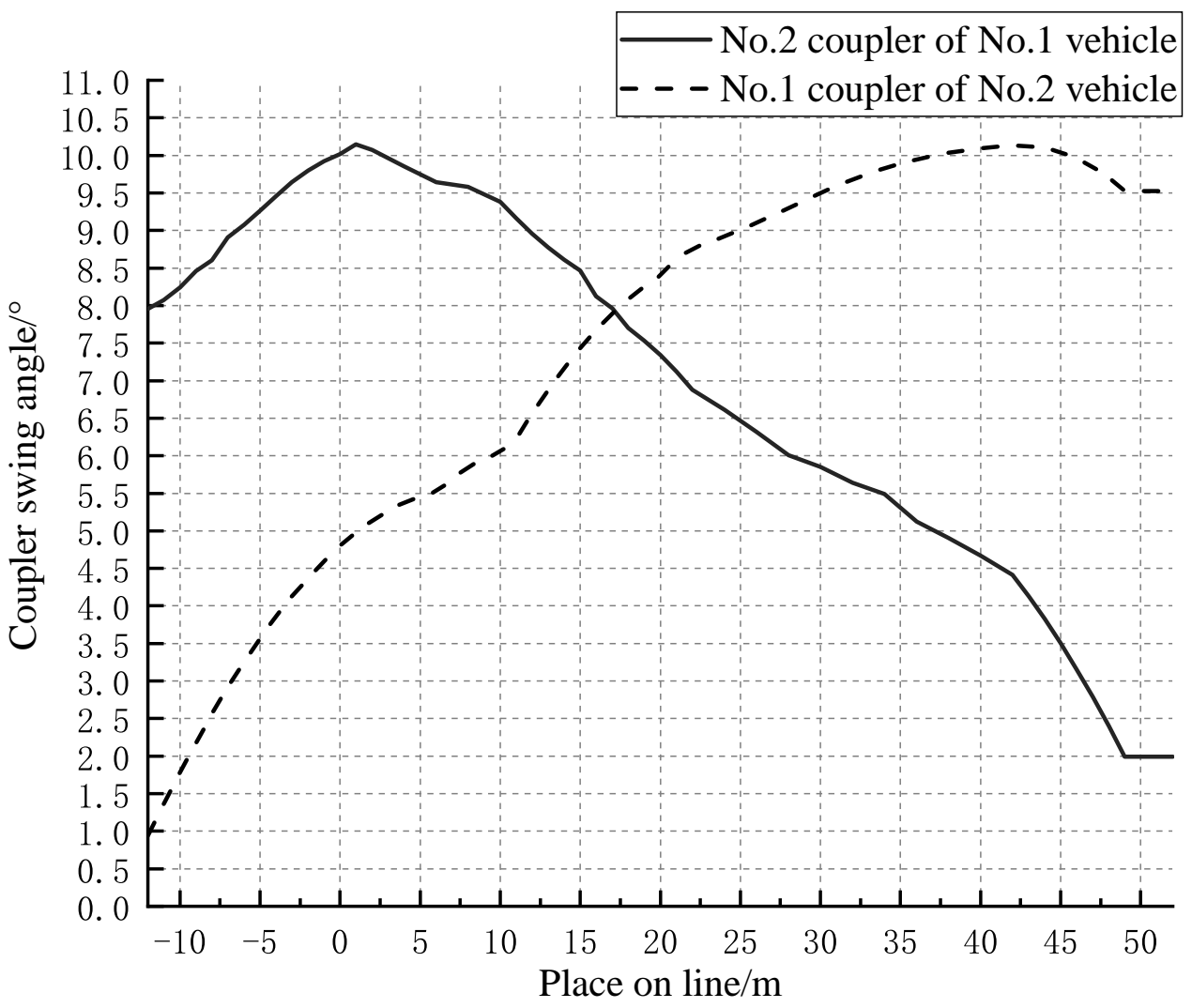

Figure 19. The variation curve of the coupler swing angle at different positions under S-curve conditions.

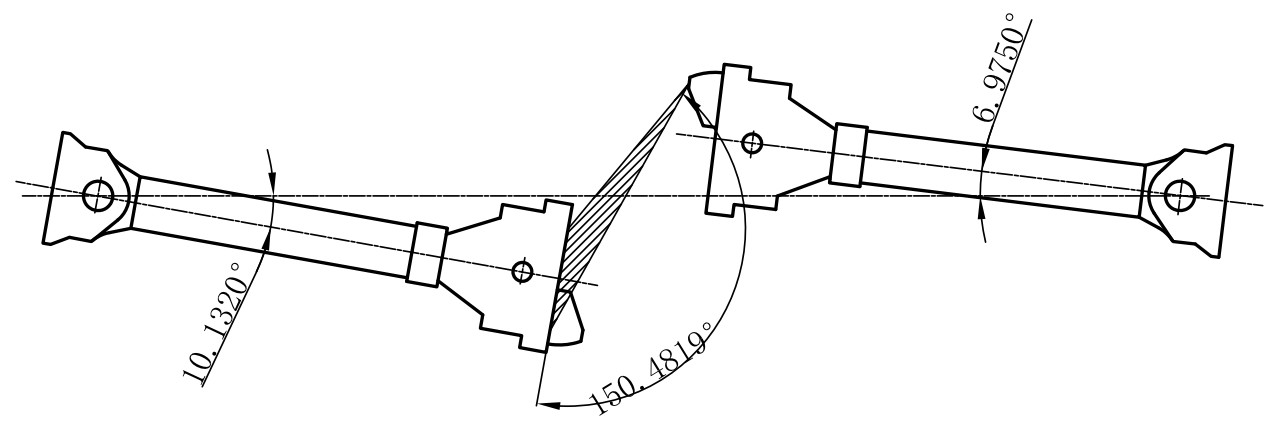

(a) $\mathrm{S} / \mathrm{N} 1$

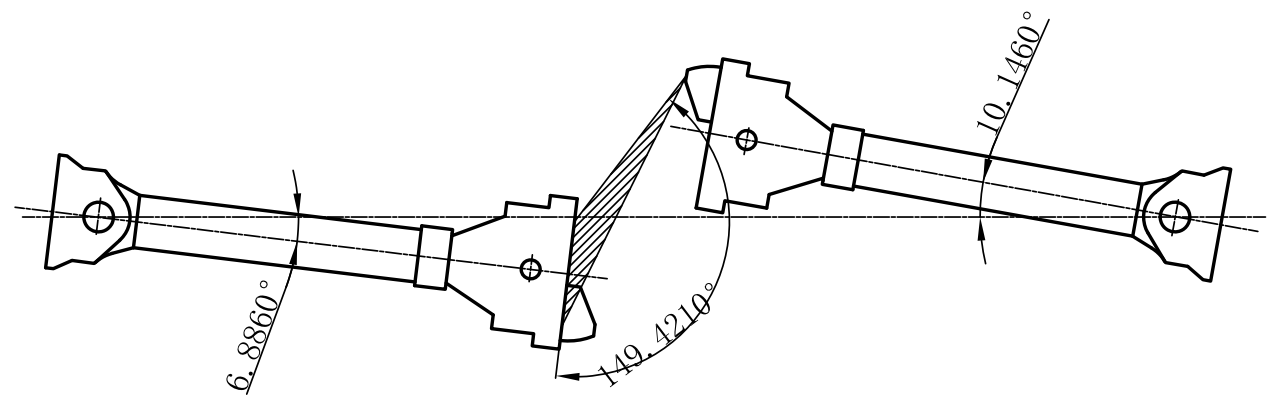

(b) $\mathrm{S} / \mathrm{N} 2$

Figure 20. The position of the hook under S-curve working condition. (a) Hook position state at the maximum swing angle of coupler No.2 of vehicle No.1 under circular S-curve working condition; (b) Hook position state at the maximum swing angle of coupler No.1 of vehicle No.2 under circular S-curve working condition. 
From this figure, it can be easily concluded that the characteristic triangle between the two vehicle hooks is an obtuse triangle. At this time, the convex cone of the front vehicle cannot be inserted into the concave cone of the rear vehicle, do not meet the requirements of safe automatic connection, i.e., the couplers cannot complete automatic connection in this working condition, and manual auxiliary connection is required.

\subsection{Data Summary}

After the calculations in Sections 5.1-5.3 and after the actual operational test of the actual vehicle on Nanchang Metro Line 4, it is concluded that: When the train runs to the circular curve-line at $445.777 \mathrm{~km} \sim 759.731 \mathrm{~km}$ on the left line of the main line, the maximum angle of the characteristic triangle of the hook is $152.1074^{\circ}$, which does not meet the requirements of automatic linkage and requires manual auxiliary linkage; When the train runs to the right line of the line $444.175 \mathrm{~km} \sim 754.205 \mathrm{~km}$ straight line into the curve-line, the maximum angle of the characteristic triangle of the hook is $167.3720^{\circ}$, at this time does not meet the automatic linkage requirements, the need for manual auxiliary linkage; When the train runs to the S-shaped curve of Wangcheng vehicle section, the maximum angle of the characteristic triangle of the hook is $150.4819^{\circ}$, which does not meet the requirement of automatic linkage and requires manual auxiliary linkage. The comprehensive data are shown in Table 5.

Table 5. The maximum angle of the characteristic triangle of the vehicle hook.

\begin{tabular}{lccc}
\hline & \multicolumn{3}{c}{ The Position of the Train on the Actual Line } \\
\cline { 2 - 5 } & $\begin{array}{c}\text { Left Line of the Main Line at } \\
\mathbf{4 4 5 . 7 7 7} \mathbf{~ k m} \sim \mathbf{7 5 9 . 7 3 1} \mathbf{~ k m}\end{array}$ & $\begin{array}{c}\text { The Right Line of the Main Line } \\
\mathbf{4 4 4 . 1 7 5} \mathbf{~ k m} \sim \mathbf{7 5 4 . 2 0 5} \mathbf{~ k m ~}\end{array}$ & Wangcheng Vehicle Section \\
\hline Maximum angle & $152.1074^{\circ}$ & $167.3720^{\circ}$ & $150.4819^{\circ}$ \\
\hline
\end{tabular}

\subsection{Train Manual Auxiliary Linkage}

When the hook span angle is too large to achieve fully automatic linkage, manual assisted linkage is required. In general, the vehicle is equipped with a special tool for manual assisted linkage-traction cable, which consists of two parts: tethering belt and tension spring. When manual assistance is needed, the traction cable pulls the head of the car hook to rotate and reach the position where it is easy to connect and move the car for connecting. To ensure the safety of the train linkage and avoid accidents.

Operation method:

- A seat (pin or bolt with holes, etc.) is added under each anti-climber at the front of the train to hold the tension spring.

- When manual assistance is required to make a linkage on a curve, observe the relative positions of the hooks of the two cars and determine in which direction the hooks need to be rotated.

- Hang the tension spring at one end of the traction cable on the seat under the anticlimber; hang the tether at the other end of the cable on the strut of the protective tread of the hook head.

- Use the fasteners on the tether to shorten the length of the tow rope and rotate the hook to the desired position for attachment. At this point the tension spring will have some extension.

- After the hook is rotated to the required position for the linkage, the personnel on the ground for manual assistance leave the linkage area; the vehicle is moved for the linkage; the hook head also oscillates to a certain extent when the linkage is made, so that the length of the extension spring changes; the traction cable is removed after the linkage is completed. 


\subsection{Symmetry Analysis}

\subsubsection{Maximum Hook Turning Angle}

Since the calculation method used by Voytek is not publicly available, this section compares the CAD 2D geometry mapping simulation analysis method with the calculation method in the text. A comparison of the maximum hook turning angles of continuous vehicles under different curve conditions is shown in Figure 21. The following conclusions can be drawn.

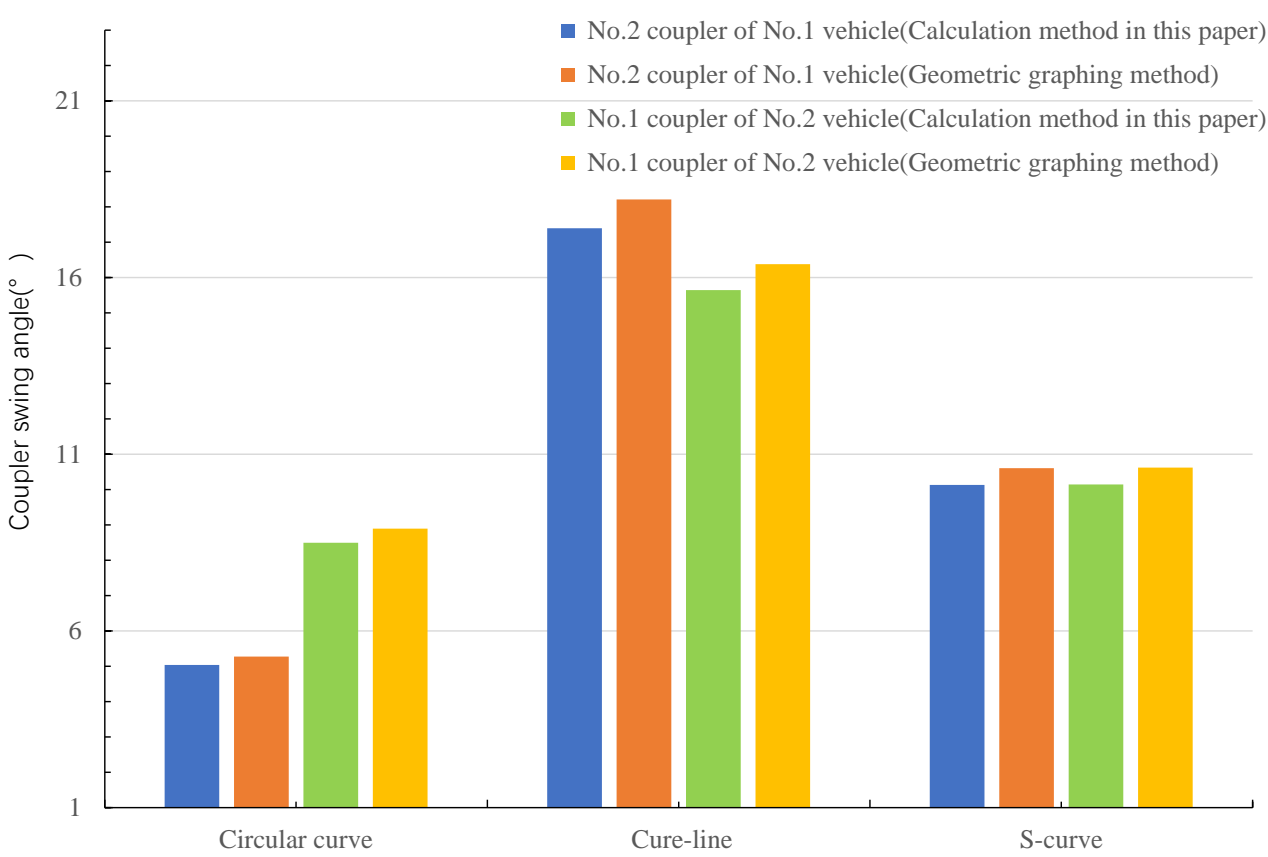

Figure 21. Comparison of maximum hook turning angle under different curve conditions.

- On the basis of the line of Nanchang Metro Line 4, the maximum hook turning angle of the same model of continuous vehicle through the horizontal curve has the largest value in the cure-line working condition, followed by the S-curve clamping straight line working condition, and finally the fixed circular curve working condition.

- The maximum hook angle derived from the simulation analysis algorithm using CAD 2D geometric mapping is slightly larger than the value derived from the graphical method, with a maximum relative error of $4.68 \%$ in the horizontal curve condition.

\subsubsection{Combined Factor Comparison}

As shown in Table 6, this section compares the three methods in terms of algorithm implementation, efficiency, economy, and stability, respectively.

Table 6. Algorithm Features.

\begin{tabular}{cccccc}
\hline & Realization Method & Efficiency & Economical & Accuracy & Stability \\
\hline CAD 2D geometric drawing method & Manual drawing & Low & Low & High & Unstable \\
Voytek's algorithm & Computer programs & High & High & Low & Stable \\
Algorithm of this paper & Computer programs & High & High & Medium & Stable \\
\hline
\end{tabular}

From the viewpoint of accuracy, the accuracy of the calculation results obtained using CAD 2D geometric drawing method is the highest; the calculation method of Voytek is less accurate because it does not consider the influence of transverse offset on the results; in this paper, the transverse offset is further considered on the basis of Voytek's algorithm, so the accuracy of our algorithm is between the two. 
However, since the CAD 2D geometric drawing method is implemented by manual drawing, the calculation efficiency of this method is low, and the stability of the calculation results is bad. Both Voytek's algorithm and the algorithm in this paper are implemented by computer programming, so both have high computational efficiency and stability of computational results.

In summary, the accuracy of our algorithm is between CAD 2D geometric mapping and Voytek's algorithm. Although its accuracy is not as high as CAD 2D geometric drawing method, the relative error of the two algorithms is only $4.68 \%$, which is within the acceptable range and improves the computational efficiency with the loss of certain accuracy.

\section{Conclusions}

To ensure the safety of the train in the curve rescue linkage operation, this paper analyzes the connection of the couplers under three different line working conditions in the context of Nanchang Railway Line 4. We propose a novel method for determining the coupler position attitude, taking into account the lateral offset of the vehicle. It is conducive to the safety of the trains when carrying out curve rescue linkage operations, and improves the calculation efficiency to a certain extent.

It is noteworthy that our calculation method compared with the traditional calculation method can be more intuitive to judge the conditions of the vehicle curve rescue linkage judgment, which is conducive to ensuring the safety of trains during curve rescue linkage operations. However, we must admit that in the above work we did not take into account the train coupling under vertical curve conditions. In future research, we need to improve our calculation method by considering the train coupling under vertical curve conditions.

Author Contributions: Conceptualization, C.Z.; Methodology, C.Z.; Visualization, C.Z.; Writingoriginal draft, C.Z.; Writing—review and editing, H.Z., F.L., X.W. and G.F. All authors have read and agreed to the published version of the manuscript.

Funding: This research was funded by the National Natural Science Foundation of China under Grant No. 51865009, National Natural Science Foundation of China under Grant No. 52065021, Jiangxi Provincial Natural Fund No.20192BAB206022.

Institutional Review Board Statement: Not applicable.

Informed Consent Statement: Not applicable.

Data Availability Statement: The data presented in this study are available on request from the corresponding author.

Conflicts of Interest: The authors declare no conflict of interest.

\section{References}

1. Pshinko, O.M.; Ursulyak, L.V.; Zhelieznov, K.I.; Shvets, A. To the problem of train running safety. IOP Conf. Ser. Mater. Sci. Eng. 2020, 985, 012014.

2. Schumann, T. Increase of capacity on the Shinkansen high-speed line using virtual coupling. In Proceedings of the COMPRAIL 2016, Madrid, Spain, 19-21 July 2016.

3. Todorov, G.; Kamberov, K.; Kralov, I.; Ignatov, I. Influence of the contact roughness upon railway monobloc wheel acoustic behaviour on virtual prototyping approach. In AIP Conference Proceedings; AIP Publishing LLC: Sozopol, Bulgaria, 7 December 2017; Volume 1910, p. 020014.

4. Magalhaes, H.; Madeira, J.; Ambrosio, J.; Pombo, J. Railway vehicle performance optimisation using virtual homologation. Veh. Syst. Dyn. 2016, 54, 1177-1207. [CrossRef]

5. Bezin, Y.; Funfschilling, C.; Kraft, S.; Mazzola, L. Virtual testing environment tools for railway vehicle certification. Proc. Inst. Mech. Eng. Part F J. Rail Rapid Transit. 2015, 229, 755-769. [CrossRef]

6. Kawasaki, K.; Seki, K. Development of a Radio Communication System Simulator for Railway Applications. Q. Rep. RTRI 2015, 56, 33-38. [CrossRef]

7. Andrew, V.; Alexander, M.; Godyaev, I. 1F11 Designing a simulation model of a railway section using CAD layers (Operation management). Jpn. Soc. Mech. Eng. 2015. Available online: https://www.jstage.jst.go.jp/article/jsmestech/2015/0/2015__1F111_/_article.html (accessed on 19 September 2021).

8. Wanchu, H. Theoretical analysis of vehicle geometry curve passage. Roll. Stock 1981, 8, 22-30. 
9. Shan, W.; Li, R. The Design and Checking Calculation of Coupled Cars in Negotiation of Curves. Roll. Stock 2004, 5, 5-8.

10. Shvets, A.O. Analysis of the dynamics of freight cars with lateral displacement of the front bogie. Adv. Math. Model. Appl. 2021, 6, $45-58$.

11. Zhang, G.; Shen, G.; Zhang, Y. The Dynamic Simulation Analysis of the Interference of the Adjacent Car-bodies and Bogies for the Dual-Hinged Train. China Railw. 2005, 4, 1-3.

12. Wang, W.; Mi, C. Parameterized calculation of geometry curve negotiation of locomotive. Railw. Locomot. Mot. Car 2007, 11, 17-20.

13. Luke, W. Calculation of curving of locomotives and vehicles by introducing the conception of equivalent radius of curve. Electr. Locomot. Mass Transit Veh. 2016, 6, 89-92.

14. Zhang, K.; Meng, L. Analysis of the curve passing capacity of the vehicle end connection device of rolling stock. China-High-Tech Enterp. 2013, 13, 103-106.

15. Li, B.; Li, D.; Ren, X.; Xu, D. Analysis on rescue of B-type metro trains connected in curve. China Railw. 2016, 06, 89-92.

16. Wang, L.; Min, Y.; Liu, X. Analysis on the Calculation Method and Result- validity of Coupling Simulations for Heavy Load Locomotive. Railw. Locomot. Car 2016, 16, 48-68.

17. Zou, R.; Ma, W.; Luo, S. Coupling stability of couplers between locomotive and vehicle. J. Traffic Transp. Eng. 2016, 16, 48-54.

18. Zhu, J. Analysis and Discussion on the Causes of the Failure of Fully Automatic Coupler. Electr. Locomot. Mass Transit Veh. 2017, 40, 90-92.

19. Ozerov, M.A.; Li, Q. Check of connected vehicles passing through the curve section. Foreign Roll. Stock 1966, 4, 50-54.

20. Zhang, P.M.; Zhang, S.H.; Jia, K. Coupling analysis on a tight-lock coupler for subway vehicle. Mach. Des. Manuf. 2011, 6, 38-40.

21. Ouyang, S.; Peng, Y.; Gao, W. Discussion of the Way to Detect the State of Coupling Structure of Couplers. Roll. Stock 2011, $49,24-28$.

22. Song, W. Study on the Distance Between Turnout and Horizontal Curve of Rail Transit in City Region. J. ECJTU 2018, 35, 22-26.

23. Li, C. Study on Minimum Curve Radius for Railway Vehicles. Railw. Stand Des. 2016, 60, 19-24.

24. Bing, L.; Ai, M.; Pan, H. Analysis of the Maximum Deflection Angle of Coupler When Railway Cars Passing Horizontal Curve. Railw. Qual. Des. 2014, 42, 3-8.

25. UIC 505-1. Railway Transport Stock-Rolling Stock Construction Ganges. 1977. Available online: https://www.docin.com/p-3498 98773.html (accessed on 20 September 2021).

26. UIC CODE 505-4. Effects of the Application of the Kinematic Gauge Defined in the 505 Series of Leafletson the Positioning of Structures in Relation to the Tracks and of the Tracks in Relation to Each Other, 4nd ed.; 2007. Available online: https://www.docin.com/p-349898 824.html (accessed on 20 September 2021).

27. Kampczyk, A. Magnetic-measuring square in the measurement of the circular curve of rail transport tracks. Sensors 2020, 20, 560. [CrossRef]

28. Kampczyk, A. Measurement of the geometric center of a turnout for the safety of railway infrastructure using MMS and total station. Sensors 2018, 20, 4467. [CrossRef] [PubMed]

29. Gu, Y.; Liu, L.; Mou, X. Main parts of the couplers for metro vehicles and their selection. Locomot. Roll. Stock Technol. 2018, 2, 34-37.

30. Xu, Z.; Ma, W.; Wu, Q.; Luo, S. Analysis of the rotation angle of a coupler used on heavy haul locomotives. Proc. Inst. Mech. Eng. Part F 2014, 228, 835-844. [CrossRef]

31. Luo, R.; Gan, F.; Teng, W.; Cong, R. Calculation of coupling vehicles geometrically passing through curves. Electr. Locomot. Mass Transit Veh. 2013, 36, 16-18.

32. Jin, X.; Zhang, H.; Shuai, G.; Kuang, X.; Lin, F. Research on Simulation Calculation of Coupler Swing Angle During Rail Vehicles Passing Curve. J. ECJTU 2019, 36, 19-24.

33. Railway Application Facilities Automatic Coupler Performance Requirements, Dedicated Interface Geometry. EN16019-2014. 2014. Available online: https:/ / www.doc88.com/p-5783803430033.html (accessed on 15 September 2021).

34. Feng, G. Analysis of the position of the center line of the coupler connection. Roll. Stock 1986, 11, 33-34. 